\title{
The Zebra Finch Paradox: Song Is Little Changed, But Number of Neurons Doubles
}

\author{
Clare Walton, Eben Pariser, and Fernando Nottebohm \\ Laboratory of Animal Behavior, The Rockefeller University, New York, New York, 10065
}

\begin{abstract}
New neurons are added to the high vocal center (HVC) of adult males in seasonally breeding songbirds such as the canary (Serinus canaria) that learns new songs in adulthood, and the song sparrow (Melospiza melodia) that does not. In both cases, the new neurons numerically replace others that have died, resulting in a seasonal fluctuation in HVC volume and neuron number. Peaks in neuronal replacement in both species occur in the fall when breeding is over and song is variable. New neurons are added, too, to the HVC of zebra finches (Taeniopygia guttata) that do not learn new songs in adulthood and whose song remains stereotyped throughout the year. Here, we show that, in contrast to the observations in seasonal songbirds, neurons added to the zebra finch HVC are not part of a replacement process. Rather, they lead to a doubling in the number of neurons that project from HVC to the robust nucleus of the arcopallium (RA). As this happens, HVC volume remains constant and the packing density of its neurons increases. The HVC-RA neurons are part of a descending pathway that carries the pattern of learned song; some HVC-RA neurons are also responsive to song playback. The addition of HVC-RA neurons happens in zebra finches housed singly, but becomes more acute if the birds are housed communally. We speculate that new neurons added to the adult HVC may help with the production or perception of learned song, or both.
\end{abstract}

\section{Introduction}

Neurogenesis in the adult avian brain was first described in the high vocal center (HVC) of the canary (Serinus canaria) song system (Goldman and Nottebohm, 1983; Paton and Nottebohm, 1984). There, new neurons numerically replace older ones of the same type that have died (Kirn and Nottebohm, 1993). The neurons that continue to be produced and replaced project from HVC to the robust nucleus of the arcopallium (RA; see Fig. 1a) and are part of the descending pathway that produces song (Alvarez-Buylla et al., 1990; Kirn et al., 1991). Electrophysiological recordings from these HVC-RA neurons in another songbird, the zebra finch (Taeniopygia guttata), suggest that some, at least, encode the pattern of learned song (Hahnloser et al., 2002).

Canaries are open-ended vocal learners that acquire their song as juveniles but then continue to add, delete, or modify syllables in their adult repertoire each year (Nottebohm et al., 1986). Recruitment of new HVC neurons fluctuates, peaking between successive breeding seasons, when the majority of the song changes also take place (Kirn

Received July 5, 2011; revised Nov. 14, 2011; accepted Nov. 22, 2011.

Author contributions: C.W. and F.N. designed research; C.W. and E.P. performed research; C.W. analyzed data; C.W. and F.N. wrote the paper.

This work was funded by the Rockefeller University. Professors Bruce McEwen and Shai Shaham from Rockefeller University and John Kirn from Wesleyan University made many helpful comments and suggestions that added to the quality of the research reported here. In addition, Professor McEwen kindly provided use of his Stereolnvestigator software. We would like to thank Dr. Nicole Creanza for editing this manuscript and providing statistics advice, Cameron Wellock for statistics advice, Sattie Haripal for immunohistochemistry advice, and Sharon Sepe for presiding over the excellent animal care program that, with the help of Helen Ecklund and Doug Yeager, made this research possible.

The authors declare no competing financial interests.

Correspondence should be addressed to Clare Walton, Flat 2 Picketts, Picketts Lane, Salfords, Surrey RH1 5RG, UK. E-mail: clareawalton@hotmail.com.

DOI:10.1523/JNEUROSCI.3434-11.2012

Copyright $\odot 2012$ the authors $\quad 0270-6474 / 12 / 320761-14 \$ 15.00 / 0$ et al., 1994). The addition of new HVC-RA neurons in adult canaries learning new songs is reminiscent of the addition of this neuron type in juvenile canaries learning their song for the first time (AlvarezBuylla et al., 1988).

Another seasonally breeding songbird, the song sparrow (Melospiza melodia), also shows seasonal peaks in new neuron addition to its adult HVC (Tramontin and Brenowitz, 1999), resulting in significant changes in neuron numbers between early spring when the birds are breeding and late autumn when breeding has ceased (Smith et al., 1997). Song sparrows only learn their song once before sexual maturity (Marler and Peters, 1987). But, as in canaries, the adult song becomes variable in the fall (Smith et al., 1997), suggesting a reduction in HVC neurons is related to a reduction in song stereotypy.

Zebra finches differ from canaries and song sparrows in that they are opportunistic breeders. Courtship in their native Australia is determined not by photoperiod, but by arrival of the rains (Zann, 1996). Male zebra finches learn their song once during juvenile development (Immelmann, 1969) and this song is retained for life with no seasonal fluctuation in stereotypy. As in canaries, zebra finches add new HVC-RA neurons during the juvenile song learning period (Nordeen and Nordeen, 1988), and this addition declines as the bird masters its song (Wilbrecht et al., 2006). Thereafter, recruitment of new HVC-RA neurons persists at a lower rate throughout adult life (Nordeen and Nordeen, 1988), diminishing with age (Wang it al., 2002). Here, we show that, in contrast to the seasonally breeding species, neurons added to the HVC of adult zebra finches do not numerically replace other neurons that have died but result in a doubling of HVC-RA neurons across adulthood.

The zebra finch has become a model for studying the basic biology of vocal learning. Our observation of a doubling in the 
number of HVC-RA neurons with modest, if any, consequences on song points to a paradox that now needs explaining.

\section{Materials and Methods}

Animals. Male adult zebra finches were used from our breeding colony at the Rockefeller University Field Research Center in Millbrook, NY. The care of all animals followed the standards set by the American Association of Laboratory Animal Care and the Rockefeller University Animal Use and Care Committee. All animals were kept on a $12 \mathrm{~h} / 12 \mathrm{~h}$ light/dark cycle with food and water provided ad libitum. Except for the "young, socially housed" birds in Experiment 3, during each experiment birds were housed alone in single cages on a rack where they could see and hear other males. Before the start of each experiment, birds were either housed with their parents and siblings in breeding cages (if 90-100 $\mathrm{d}$ old at the experiment start) or in larger stock cages containing other males (if $>100 \mathrm{~d}$ old at the experiment start). For the older birds, a detailed history is not available. It is most likely that they were used for breeding as younger adults and then retired into male stock cages.

Three experiments. Experiment 1 used 34 adult males ranging in age between $90 \mathrm{~d}$ and 11 years to look for changes in the number and density of different HVC neurons with age. These birds were also used to measure neuron density in an area of the caudal nidopallium (NC) under HVC. Experiment 2 used 40 adult males to test how HVC-RA neurons born in juveniles and adults survived during the first year of life and, in six additional birds, during the first 4 years of life. Experiment 3 used 26 adult males to look at the effects of age and social environment on the survival of adult-born neurons in HVC.

Surgery and birthdate labeling. The 34 birds in Experiment 1 were anesthetized with Nembutal (pentobarbital sodium, $60 \mathrm{mg} / \mathrm{kg}$; Ovation Pharmaceuticals) and injected into both hemispheres with Cholera toxin B (CTB) conjugated to AlexaFluor dyes (1\% diluted in sterile saline; Invitrogen). Half of these birds received injections to both nucleus RA and Area X (Fig. 1b) in the same surgery: green Alexa-488-CTB to RA and red Alexa-594-CTB to Area X. The remaining 17 birds only received injections of green Alexa-488-CTB to nucleus RA to add more time points to the analysis of HVC-RA neurons. Six days were allowed for retrograde transport of the CTB, and then the birds were anesthetized and killed by intracardial perfusion of saline and $4 \%$ paraformaldehyde (PFA).

Forty of the 46 birds in Experiment 2 were given, starting at 90-98 d of age, three consecutive days of 5-bromo-2-deoxyuridine (BrdU) injections ( $100 \mu \mathrm{l}$ of $10 \mathrm{mg} / \mathrm{ml} \mathrm{BrdU}$ dissolved in sterile saline; $\sim 80 \mathrm{mg} / \mathrm{kg}$ for a typical $12 \mathrm{~g}$ bird) into the breast muscle twice a day at 8:00 A.M. and 8:00 P.M. On the fourth day, these birds were anesthetized with Nembutal and given bilateral injections of red latex microspheres (Lumafluor) into RA (40 $\mathrm{nl}$ per hemisphere). In this experiment, fluorescent latex microspheres were used as the retrograde tracer because microsphere labeling persists for at least a year in vivo (Katz et al., 1984) and they have previously been used to show loss of HVC-RA neurons in the canary (Kirn and Nottebohm, 1993). The birds were randomly divided into four groups of 10 birds each and killed 1,3,6, or 9 months later by intracardial perfusion of saline and 4\% PFA. A fifth group of six birds, 90-95 d old, was given $3 \mathrm{~d}$ of $\mathrm{BrdU}$ injections without the microsphere injection surgery and then killed 4 years later by intracardial perfusion. Six days before death, this group received, under Nembutal anesthesia, bilateral injections of green CTB into RA to enable the identification of BrdU-positive HVC-RA neurons.

Experiment 3: The effect of a complex social environment on the survival of adult-born neurons in HVC was tested in 14 adult males. Starting at 90-99 d of age, these birds received three consecutive days of BrdU injections, as above, and $3 \mathrm{~d}$ later were moved to a large aviary $\left(5^{\prime} \times 6^{\prime} \times 2^{\prime}\right)$ containing 14 other males and 15 females. These young, socially housed males were randomly split into two groups and killed either 1 or 3 months after the last day of BrdU injections. To look for survivorship of HVC neurons in older adults, 12 birds between 2 and 2.25 years of age received BrdU injections, as above, and were killed either 1 or 3 months later. These "old, singly housed " birds were housed in single cages on a rack where they could see and hear other males for the duration of the experiment, in exactly the same manner as the young birds in Experiment 2. Overall, Experiment 3 compared the survival of adultborn neurons at 1 and 3 months between three different conditions: young, socially housed; old, singly housed; and the "young, singly housed" group from Experiment 2.

Tissue processing and microscopy. Brains from birds in all three experiments were removed after perfusion, postfixed in $4 \%$ PFA, and cryoprotected through ascending concentrations of sucrose in PBS $(5,15$, and $30 \%$ ) for $2 \mathrm{~d}$ at $4^{\circ} \mathrm{C}$. Brains were frozen in Neg-50 frozen section medium (Richard Allan Scientific) and frontally sectioned at $40 \mu \mathrm{m}$ on a cryostat. Sections containing HVC were collected in three series, each containing every third section, to allow more than one staining procedure to be performed for each bird. After the appropriate staining procedure, a confocal Z-stack of every third section was taken, using a $20 \times / 0.75 \mathrm{NA}$ objective lens on a Zeiss inverted LSM 510 META laser-scanning confocal microscope, and used to count cells. Confocal stacks contained either two or three color channels depending on the particular experiment. HVC sections were also imaged on an Olympus IX70 inverted microscope using a $10 \times / 0.3 \mathrm{NA}$ objective lens to provide $10 \times$ images for measuring the volume of HVC.

For birds in Experiment 1, one set of HVC slides was used to count the two kinds of retrogradely labeled projection neurons (HVC-RA and HVC-X). Mounted sections were stained with $1 \mu \mathrm{M}$ ToPro-3-iodide (Invitrogen) to identify cell nuclei. Confocal Z-stacks of HVC contained the following three color channels: the green CTB retrograde label from RA, the red CTB retrograde label from Area X, and the far-red ToPro signal. A subset of 16 birds was selected, spanning the age range, and a second set of their HVC-containing slides was stained with an antibody against the neuronal marker Hu. Z-stacks collected through HVC contained the far-red Hu staining and the green CTB retrograde label from RA, which was used to define the boundary of HVC during counting. A second Z-stack with the same two color channels was taken of an area of NC below HVC in the eight most rostral sections of the series to allow counting of $\mathrm{Hu}$-positive neurons in NC (see Fig. 1 for area sampled).

For the 46 birds in Experiment 2, one set of HVC slides was stained with antibodies against $\mathrm{BrdU}$ and $\mathrm{Hu}$. Confocal Z-stacks were collected containing these two antibody signals plus the retrograde tracer label from RA, which was red microspheres for the 40 birds killed after 1, 3, 6, or 9 months and green CTB for the six birds killed after 4 years. In these six birds, a second set of HVC-containing slides was stained for the combination of BrdU and three interneuron markers to look for the presence of adult-born interneurons in HVC. Every confocal stack was examined for the presence of BrdU-positive nuclei in the center of a cytoplasm that was positive for the mix of interneuron antibodies. Both 
left and right HVC was examined for each bird. For the 26 birds in Experiment 3, one set of HVC slides was stained with antibodies against BrdU and $\mathrm{Hu}$ and imaged using two-color confocal microscopy.

Immunohistochemistry. Sixteen of the birds in Experiment 1 had a series of HVC slides stained with an antibody against $\mathrm{Hu}$ as follows: mounted sections were incubated in citrate buffer $(0.01 \mathrm{M}$ sodium citrate plus $0.01 \mathrm{M}$ citric acid) at $37^{\circ} \mathrm{C}$ for $1 \mathrm{~h}$ followed by $0.125 \%$ pepsin in $0.1 \mathrm{~N}$ $\mathrm{HCl}$ for $3 \mathrm{~min}$. Slides were then blocked in $10 \%$ normal goat serum in $0.3 \%$ Triton X-100 and sequentially incubated with mouse anti-Hu primary (anti-HuC/D 16A11, Invitrogen) at 1:150 in blocking solution for $48 \mathrm{~h}$ at $4^{\circ} \mathrm{C}$ and Alexa Fluor 647 goat anti-mouse secondary IgG (Invitrogen) at 1:500 for $2 \mathrm{~h}$ at room temperature.

All birds in Experiments 2 and 3 had a series of HVC sections stained with the combination of antibodies against $\mathrm{BrdU}$ and $\mathrm{Hu}$ as follows: mounted sections were incubated in $60 \%$ formamide at $55^{\circ} \mathrm{C}$ for $15 \mathrm{~min}$, washed in TBS (Tris-buffered saline, $\mathrm{pH} 7.5$ ) for $10 \mathrm{~min}$, incubated in $2 \mathrm{~N}$ $\mathrm{HCl}$ at $37^{\circ} \mathrm{C}$ for $30 \mathrm{~min}$, incubated in sodium borate solution $(0.1 \mathrm{M}$ $\mathrm{NaB}_{4} \mathrm{O}_{7}, \mathrm{pH}$ 8.5) for 10 min, washed three times in TBST (TBS containing $0.1 \%$ Triton $\mathrm{X}$ ), blocked with $10 \%$ normal goat serum in TBST for 30 min at room temperature, and then incubated with the anti-Hu antibody at 1:150 in TBST with $10 \%$ normal goat serum for $48 \mathrm{~h}$ at $4^{\circ} \mathrm{C}$. On day 3 , mounted sections were washed three times in TBST for 10 min each and incubated with Alexa-647 conjugated goat anti-mouse secondary antibodies at 1:500 in TBST for $2 \mathrm{~h}$ at room temperature. Sections were washed three times in TBS for 10 min each, blocked with $10 \%$ normal goat serum in TBST for $30 \mathrm{~min}$ at room temperature, and incubated with anti-BrdU (rat monoclonal primary, Axyll) at 1:500 in TBST with $10 \%$ goat serum overnight at $4^{\circ} \mathrm{C}$. On day 4 , sections were washed three times in TBS for 10 min each and incubated with either Alexa- 488 or Alexa-555 conjugated goat anti-rat secondary at 1:500 in TBST for $2 \mathrm{~h}$ at room temperature.

The six 4-year survival birds from Experiment 2 had one series of HVC-containing slides stained for three calcium-binding proteins known to label HVC interneurons: parvalbumin (PV), calbindin (CB), and caltretinin (CR). Since HVC interneurons often express more than one of the three calcium-binding proteins (Wild et al., 2005), we combined the primary antibodies against all three proteins and used the antibody mix to label neurons expressing one or more of these markers. Scotto-Lomassese et al. (2007) showed that this approach labels the same total number of neurons as can be labeled by both GABA and $\mathrm{Hu}$ together (i.e., GABA-positive neurons), and thus, it is likely that using a combination of antibodies for PV, CB, and CR together identifies the entire interneuron population of HVC. Primary antibodies against the marker proteins were first tested individually in $40-\mu \mathrm{m}$-thick mounted sections of adult zebra finch tissue to ensure good antibody penetration. The antibodies used were mouse anti-PV, rabbit anti-CB, and goat anti-CR (all from Swant). Once each staining had been optimized, all three primaries were combined into a single primary antibody mix for use on the HVC sections of the 4-year survival Experiment 2 birds. The final protocol was as follows: mounted sections were incubated in $0.2 \%$ PBST (PBS with $0.2 \%$ Triton $\mathrm{X}$ ) for $1 \mathrm{~h}$ at room temperature, blocked with $4 \%$ BSA in $0.2 \%$ PBST for $1 \mathrm{~h}$ at room temperature, and then incubated at $4^{\circ} \mathrm{C}$ for $72 \mathrm{~h}$ with the following mix of primary antibodies: mouse anti-PV, rabbit anti-CB, and goat anti-CR, each used at 1:5000 in blocking solution. Slides were washed in PBS and incubated for $2 \mathrm{~h}$ at room temperature with the following mix of secondary antibodies: Alexa-647 goat anti-mouse, Cy5 goat anti-rabbit, and Alexa-647 donkey anti-goat, each used at $1: 500$ in $4 \%$ BSA in $0.2 \%$ PBST. Slides were washed in PBS, fixed in 4\% PFA for 10 min, washed again in PBS, incubated in $2 \mathrm{~N} \mathrm{HCl}$ at $37^{\circ} \mathrm{C}$ for $30 \mathrm{~min}$, incubated in sodium borate solution (0.1 M NaB4O7 pH8.5) for $10 \mathrm{~min}$, washed again in PBS, and blocked with $10 \%$ normal goat serum in $0.2 \%$ PBS for $1 \mathrm{~h}$ at room temperature. Slides were incubated sequentially with the rat anti-BrdU primary at 1:500 in blocking solution overnight at $4^{\circ} \mathrm{C}$ and Alexa-555 goat anti-rat secondary at 1:500 in PBS for $2 \mathrm{~h}$ at room temperature.

Neuron counts. Counts of HVC-RA and HVC-X projection neurons and $\mathrm{Hu}$-positive neurons were all performed using the optical fractionator method (West and Gundersen, 1990) in Z-stack images taken on a confocal microscope and using StereoInvestigator software with the Im- age Stack Module (MBF Biosciences). Briefly, a grid of regularly spaced counting squares was placed on top of the confocal stack and only squares falling within the area of interest (HVC or NC under HVC) were used to count cells. Each square was made into a 3D counting box by adding a box height in the $z$-axis that was shorter than the thickness of the mounted tissue section. Cells were counted if their nucleus (either stained with a nuclear stain or the dimly stained center of a Hu stained neuron) was within a counting box and if they met the staining criteria defined for each neuron type (see below). Two edges of the counting box were "exclusion edges" and any cell nuclei touching these edges were excluded from counts. Nuclei touching or straddling the other two edges were included. A 3- $\mu$ m-thick guard layer was applied to the top and bottom of the Z-stack and cells were excluded from the count if the broadest cross-section of their nucleus was in focus within either guard layer. This eliminates the possibility of a cell being represented twice in adjacent sections, and so no corrections for cell splitting errors were required to estimate the packing density or total number of cells in the area of interest. The final sampling grid square size (i.e., the distance between counting boxes) and the counting box dimensions were chosen to give counts of $\sim 400$ cells per HVC for both RA-projecting and Hupositive neurons and $\sim 150$ cells per HVC for X-projecting neurons, which are much scarcer. The counting box height was adjusted for each cell type to ensure that cell counts were only performed in tissue with full antibody penetration. Final dimensions were as follows: for HVC-RA and HVC-X neurons, grid square size $=150 \mu \mathrm{m}$, counting box X/Y dimension $=50 \mu \mathrm{m}$, and counting box height $=20 \mu \mathrm{m}$; for Hu-positive neurons, grid square size $=100 \mu \mathrm{m}$, counting box X/Y dimension $=25$ $\mu \mathrm{m}$, and counting box height $=16 \mu \mathrm{m}$.

In Experiment 1, HVC-RA and HVC-X projection neurons were identified by the presence of either green or red CTB, respectively, rimming a nucleus stained with ToPro- 3 with a diameter $>4 \mu \mathrm{m}$. This cutoff was selected by measuring the diameter of 20 HVC-RA neurons per HVC in the Experiment 1 birds and finding that no complete neuron had a nuclear diameter smaller than $4 \mu \mathrm{m}$. Thus, nuclei smaller than this cutoff were taken to be cells fragmented by sectioning. If a continuous area of CTB was touching two nuclei, only one nucleus was counted as a positive backfilled cell. Hu-positive neurons were identified as having $\mathrm{Hu}$ staining in a circular pattern typically with a dim region in the center to mark the nucleus. In Experiment 2, HVC-RA neurons were identified by the presence of punctate red microspheres clustered in a Hu-positive cytoplasm around a dimly or negatively stained nucleus. For HVC-RA and HVC-X projection neuron counts in most birds, the left and right HVC were analyzed independently and then averaged. For a small minority of birds, only one HVC was available due to an incomplete labeling with retrograde tracer or to bad tissue processing. In these cases, the data from a single $\mathrm{HVC}$ is presented as we found no systematic differences between left and right HVC. For Hu-positive neuron counts in both HVC and NC, one hemisphere, selected at random, was analyzed per bird. Again, there were no systematic differences between left and right counts in either brain region.

When using the optical fractionator method in tissue sections, final cell counts refer to the total number of cells in the region of interest. Our confocal stacks did not cover the entire cross-sectional area of either HVC or NC (Fig. 1c), so the final cell counts only corresponded to the volume of each brain area included in the confocal stacks. For both HVC and $\mathrm{NC}$, we measured this volume by summing the cross-sectional area contained within each stack and multiplying the total area by the section thickness and sampling interval $(40 \mu \mathrm{m} \times 3)$. Neuron counts were then expressed as the number of neurons per unit volume, i.e., neuron density. For HVC, the total number of each neuron type was estimated by multiplying neuron density by the total volume of HVC, which was measured in $10 \times$ images using ImageJ software (NIH, Bethesda, MD). The boundaries of HVC were defined by the retrograde label from RA in Experiments 1 and 2 and the characteristically large Hu-positive soma of the HVC-X neurons in Experiment 3. Since NC does not have discrete anatomical boundaries on all its sides, we were not able to get an estimate of total NC neuron numbers and in this case we settled just for the packing density of neurons in the region of $\mathrm{NC}$ we sampled. 
In Experiments 2 and 3, BrdU-positive neurons were counted using a modified version of the optical fractionator method where, instead of using a sampling grid, the entire area of interest within the confocal stack was scanned for positive neurons. A guard volume of $3 \mu \mathrm{m}$ was still applied to the top and bottom of the Z-stack to ensure split cells were not counted. BrdU/Hu double-positive neurons were counted if they had a bright BrdU-positive nucleus (either red or green) within an area of Hu-positive cytoplasm. In the 40 Experiment 2 birds that were killed after $1,3,6$, or 9 months, triple-labeled $\mathrm{BrdU} / \mathrm{Hu} / \mathrm{microsphere-positive}$ neurons were counted if they had punctate red microspheres clustered around a green BrdU-positive nucleus in the center of a $\mathrm{Hu}$-positive cytoplasm. For the six 4 -year survival Experiment 2 birds, BrdU/Hu/CTB triple-labeled neurons were counted if there was green CTB rimming a red BrdU-positive nucleus in the center of a Hu-positive cytoplasm (see Fig. $6 a$ ). Both the density and total number of BrdU-positive neurons in HVC were calculated, as described above for the projection neurons.

Due to the high degree of variability in HVC volume between individual birds (Fig. 2b), counts of each HVC neuron type at a given age show more variability than the packing density measurements for that neuron type. For that reason, the present report emphasizes neuron packing density rather than total neuron number. However, because variability in HVC volume (and therefore in total neuron number) may be biologically relevant, statistical analyses were conducted for both neuron density and total neuron number for all counts presented here. All significant relationships described for neuron density also apply to changes in total neuron numbers.

Error of cell counts. To determine the precision of the cell counting procedure, the Gunderson coefficient of error (CE) was calculated for each HVC in the sample. Previous analyses in biological structures have indicated that a $\mathrm{CE}$ of 0.1 or lower is adequate to find a real difference in the number of objects counted between two different samples (Gundersen and Jensen, 1987; West, 1993), and thus we modified our grid and counting box dimensions to ensure CE values were lower than 0.1 in all experiments.

Nucleus diameter measurements. To assess the nuclear diameters of HVC-RA neurons in birds from Experiment 1, the confocal stacks from three left and three right HVC sections were chosen from each bird and a single image from the center of each stack was analyzed using ImageJ software. From this material, 20 CTB-labeled HVC-RA neurons were selected from each HVC (spread across the three images) and the diameter across the widest dimension of its ToPro-positive nucleus was measured. Since we wanted to measure the true diameter of nuclei, we selected profiles with the broadest cross sections. The 40 diameters from the left and right HVCs were combined to find the average nuclear diameter for each bird. There was no systematic difference between the mean HVC-RA nuclear diameters from the left and right hemispheres.

Ventricular zone cell counts. The number of BrdU-positive cells residing in the ventricular zone (VZ) above HVC was counted in the birds from Experiments 2 and 3 . These cells were counted in every third $40 \mu \mathrm{m}$ section using the same confocal stacks that were used to count BrdU/Hu doublepositive HVC neurons. Positive VZ cells were identified by the presence of a BrdU-labeled nucleus that was negative for $\mathrm{Hu}$ staining and was touching the ventral ventricular surface above HVC (see Fig. 8a). The length of the VZ present in each confocal stack was traced in StereoInvestigator software (MBF Biosciences). Total counts were converted to counts per square millimeter of VZ by dividing them by the total VZ that had been available to count, which was determined by summing the VZ lengths from every counted section and multiplying it by the section thickness $(40 \mu \mathrm{m})$. For each bird, the counts from the left and right VZs were averaged.

Modeling the net addition of new neurons to HVC. In 2002, Wang et al. published data showing how the addition of new HVC-RA neurons changes with age in the adult zebra finch. This paper found that the number of ${ }^{3} \mathrm{H}$-thymidine/fluorogold double-positive HVC-RA neurons surviving for 4 months in HVC declines with the age at which ${ }^{3} \mathrm{H}$ thymidine was administered. The data points were extracted from the paper and nonlinear regression was performed in Matlab 6.1 (The MathWorks) to find a rate for the decline. The following exponential decay equation provided the best fit: $R_{t}=111 e^{-0.002 t}$.

In this equation, $t$ is the bird's age in days and $R_{t}$ is the number of HVC-RA neurons added per day at $t$ days of age. This equation describes
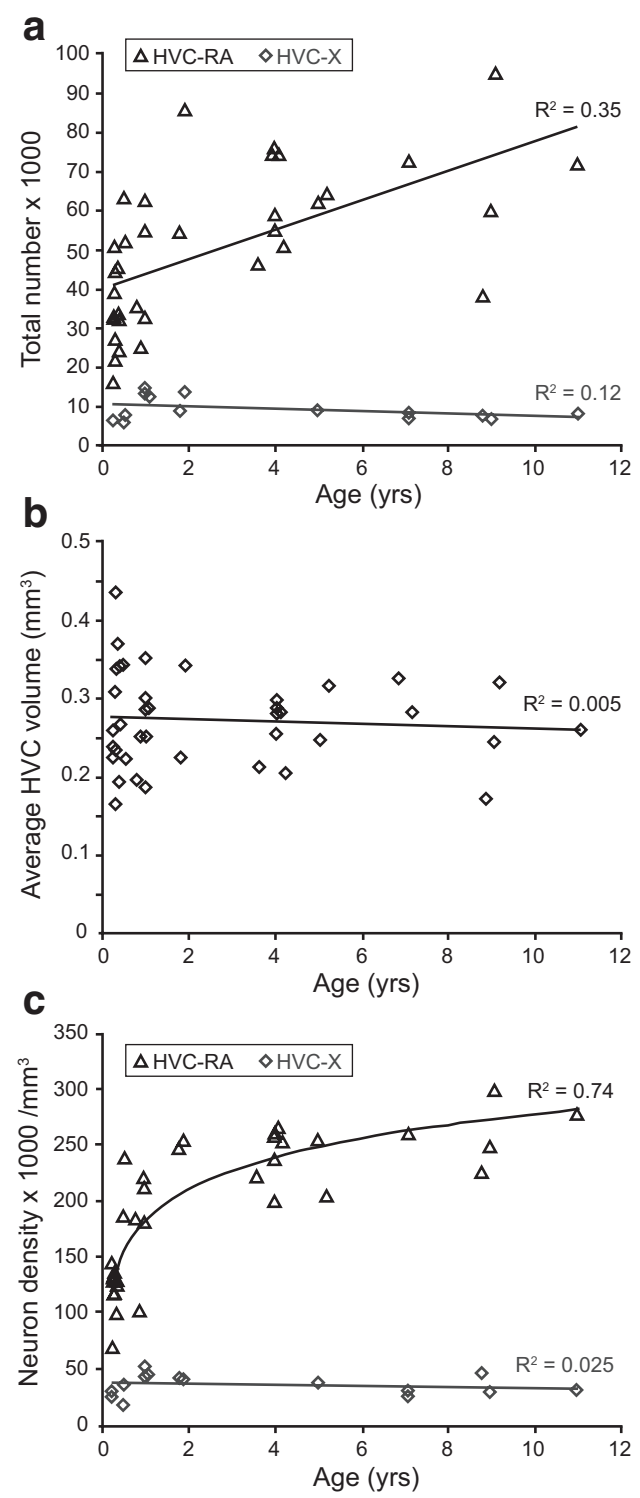

Figure 2. The total number and packing density of HVC-RA neurons increases between $90 \mathrm{~d}$ and 11 years of age in the adult malezebra finch whereas the number and density of HVC-X neurons do not change over the same age range. $\boldsymbol{a}$, There is a significant increase in the total number of HVC-RA projection neurons between $90 \mathrm{~d}$ and 11 years of age $(p<0.0005)$, but there is no change in the number of HVC-X projection neurons over the same age range $(p=0.19)$. $\boldsymbol{b}$, HVC volume does not change with age between $90 \mathrm{~d}$ and 11 years $(p=0.67) . c$, The packing density of HVC-RA neurons in HVC significantly increases between $90 \mathrm{~d}$ and 11 years of age in the adult male zebra finch and this relationship is best modeled using logarithmic regression $\left(p=1.8 \times 10^{-10}\right)$. The density of HVC-X neurons does not change with age overthesame age range $(p=0.56)$. For all threegraphs, each data point represents the average of the left and right HVC from one bird.

the number of new HVC-RA neurons that, at a given bird age, will be added to HVC each day and will survive for 4 months. Integrating this rate equation between two particular time points gives the total number of HVC-RA neurons added between those time points. If we make the assumption that every new HVC-RA neuron that survives for 4 months will survive indefinitely, we can integrate the rate equation to model how the total population of HVC-RA neurons would change with age if the assumption were correct:

$$
N_{i}=\left(\int_{90}^{i} 111 e^{-0.002 t} d t\right)+c .
$$

The integration was performed between day 90 and day $i$, where day $i$ is the age of the bird in days, because we only wanted to model the process 
of neuronal addition in the adult bird. We take $90 \mathrm{~d}$ of age to represent the start of adulthood in zebra finches. In this equation, $N_{i}$ is the total number of HVC-RA neurons present in a bird $i$ days old (as long as $i$ is $>90 \mathrm{~d}$ ). The variable $c$ is the number of HVC-RA neurons present in a 90-d-old male before any adult-born neurons have been recruited, and $t$ represents time in days. We calculated a value for $c$ by finding the average number of HVC-RA neurons present in our 90-d-old experimental birds and then used this value to derive a set of predicted values for $N_{i}$ at ages ranging from $91 \mathrm{~d}$ to 11 years. This set of predicted values shows how the total HVC-RA neuron number would change with age if we were correct in assuming that every new neuron surviving in HVC for 4 months survives indefinitely. The predicted values for total HVC-RA number were converted to HVC-RA density by dividing them by $0.27 \mathrm{~mm}^{3}$, the mean HVC volume across all 34 experimental birds. The predicted HVC-RA density values were then plotted on the same graph as the observed HVC-RA density data (see Fig. 4). To determine how accurately the predicted values fit the observed data, we calculated the sum of squares of the residuals between the model and the observed data and used these to calculate an $R$-squared value.

Statistics. All statistical tests were performed using SPSS 16.0. For the counts in Experiment 1, linear regression was performed to calculate the $R$-squared and $p$ values for each dataset. For the HVC-RA density data, regressions were performed using linear, logarithmic, and exponential models and the best fit equation, in this case a logarithmic model, was chosen to generate an $R$-squared and $p$ value for the fit. For Experiment 2 , neuron counts (total numbers and neuron densities) across the multiple survival times were compared using one-way ANOVAs. For Experiment 3 , $\mathrm{Hu} / \mathrm{BrdU}$ neuron counts from the three different conditions (young, singly housed; young, socially housed; old, singly housed) were compared using an unweighted-means two-way ANOVA. An unweighted-means analysis was performed because there was a large difference in number of birds in each group that caused heterogeneity of variance between the three groups. BrdU-positive ventricular zone cells in all three groups were compared using a weighted-means two-way ANOVA followed by the Tukey HSD post hoc test.

\section{Results}

\section{Experiment 1: Net addition of HVC-RA neurons in the adult zebra finch}

There are two known populations of projection neurons in HVC: the HVC-RA neurons that are continuously produced in adulthood, and a second type that send projections down to Area X in the medial striatum (HVC-X neurons), which are mainly produced in the embryo or the first few days after hatching (Alvarez-Buylla et al., 1988; Scotto-Lomassese et al., 2007). Using fluorescently labeled cholera toxin B (CTB) as a retrograde tracer, we labeled these two neuron populations with different color dyes (Fig. 1a) and counted the number of each neuron type in adult male zebra finches, ranging in age from $90 \mathrm{~d}$ to 11 years, to see if the numbers of either neuron type changed with age. There was a significant increase in the total number of retrogradely labeled HVC-RA neurons resulting in a doubling of their mean number between $90 \mathrm{~d}$ and 11 years of age (Fig. $2 a$; linear regression: $R^{2}=0.35, p<$ $0.0005)$. In contrast, the number of HVC-X neurons showed no change over the same span of years (Fig. $2 a$; linear regression: $\left.R^{2}=0.12, p=0.19\right)$. Thus, a net increase in the number of HVC-RA neurons retrogradely labeled in adulthood was specific to the neuron type known to be produced in the adult brain.

We measured the volume of HVC to see whether it grew to accommodate the extra HVC-RA neurons but found that the mean HVC volume did not change between $90 \mathrm{~d}$ and 11 years of age (Fig. $2 b$; linear regression: $R^{2}=0.005, p=0.67$ ). There was, however, a considerable degree of heterogeneity in HVC volume between individuals of a given age. This variability has been described by others for both canaries (Nottebohm, 1981) and zebra finches (Airey et al., 2000). Since variation in HVC size was a significant source of scatter in our total neuron counts, we also calculated the density of HVC-RA and HVC-X neurons for each bird by dividing neuron numbers by the volume of each HVC. The neuron density data showed less variability than total neuron number. It revealed a highly significant increase in the density of HVC-RA neurons between $90 \mathrm{~d}$ and 11 years of age that was described better by a logarithmic relationship than by a linear one (Fig. $2 c$; log regression: $R^{2}=0.74, p=1.8 \times 10^{-10}$; linear regression: $\left.R^{2}=0.56, p=0.00001\right)$. A logarithmic relationship indicates that the rate of increase slows with age, presumably as the rate of neuronal addition to HVC also slows (Wang et al., 2002). In accordance with the total HVC-X neuron numbers and the observation that no new HVC-X neurons are produced in adulthood (Scotto-Lomassese et al., 2007), there was no change in the density of HVC-X neurons across the adult lifespan (Fig. $2 c$; linear regression: $R^{2}=0.03, p=0.56$ ). Thus, while the HVC-X neuron population remains fixed with age, new adult-born HVC-RA neurons continue to be added within the existing HVC volume.

The observed increase in retrogradely labeled HVC-RA neurons with age could have resulted from an increase in the efficiency of neurons to take up retrograde tracer with age, rather than a genuine increase in the total number of neurons. To test for this possibility, we stained HVC sections with an antibody against $\mathrm{Hu}$, a marker of neuronal identity that has been previously tested in the songbird brain (Barami et al., 1995). We counted the number of $\mathrm{Hu}$-positive HVC neurons in birds ranging in age between $90 \mathrm{~d}$ and 9 years. Since HVC-RA neurons make up approximately half of all the neurons in HVC (Nordeen and Nordeen, 1988; Kirn et al., 1991; Ward et al., 2001), a genuine increase in the number of HVC-RA neurons should also be reflected in the total neuron population of HVC. Indeed, there was a significant increase in both the total number and the packing density of Hu-positive neurons in HVC with age between $90 \mathrm{~d}$ and 9 years (Fig. $3 a$; linear regression for neuron density: $R^{2}=$ $0.63, p=0.0002$; linear regression for total neuron number: $R^{2}=$ $0.28, p=0.036$ ). Moreover, the increase in Hu-positive HVC neurons was on the order of magnitude that we would expect given that HVC-RA neurons make up half of the total population; the packing density of HVC-RA neurons doubled between $90 \mathrm{~d}$ and 9 years while that of $\mathrm{Hu}$-positive neurons increased by $\sim 50 \%$ over this time. Thus, the observed increase in HVC-RA neurons reflected a true increase in the number of neurons and not a change in efficiency of retrograde transport with age. We do not know why the total number of HVC neurons increased linearly with age (Fig. $3 a$ ), while that of HVC-RA neurons increased in a nonlinear manner (Fig. 2c). Perhaps, new HVC-RA cells turn on the expression of $\mathrm{Hu}$ before they are able to transport retrograde tracer from RA and thus, because more new HVC-RA neurons are produced in younger adults, counts of retrogradely labeled neurons underestimates the true number of HVC-RA cells present in this age group.

Since previous work in the canary found that the cell nucleus, and thus presumably the cell soma, of adult-born HVC neurons got smaller as the neurons aged (Kirn et al., 1991), we wondered whether the increase in HVC-RA density with age in the adult zebra finch was also accompanied by a shrinking of the HVC-RA neurons. To get an indication of HVC-RA cell size, we measured the nuclear diameter of 40 HVC-RA neurons per bird (20 from each HVC) across the range of bird ages from $90 \mathrm{~d}$ to 11 years. There was a reduction in the mean nucleus diameter of the HVC-RA neurons from $6.5 \mu \mathrm{m}$ in $90 \mathrm{~d}$ olds to $5.4 \mu \mathrm{m}$ in older adults (7-11 years old), and the decrease in nucleus size was linear 


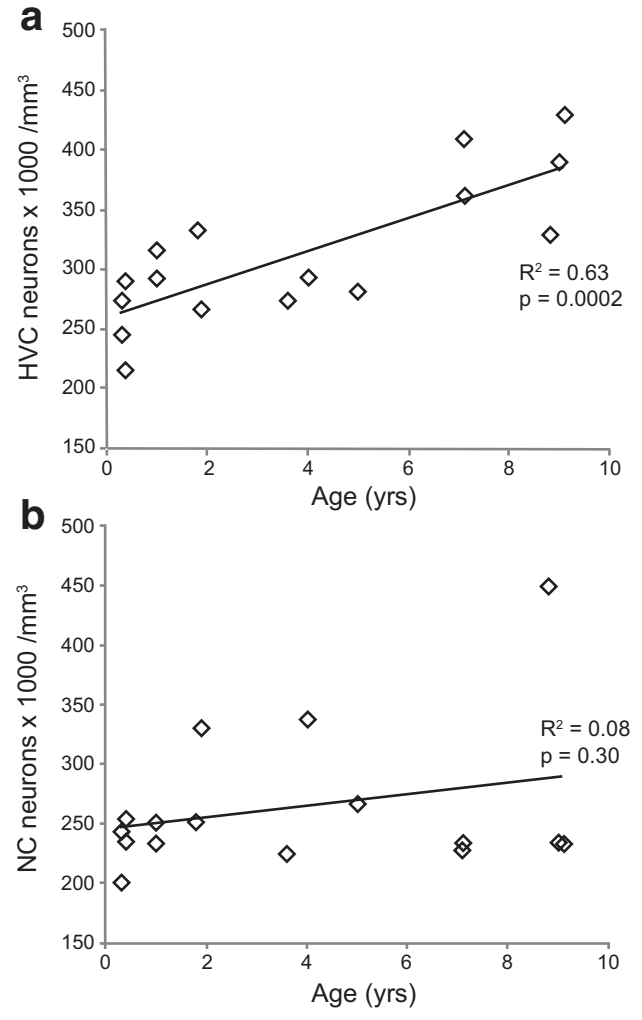

Figure 3. The packing density of Hu-positive neurons significantly increases with age in the HVC, but not in an area of NC ventral to HVC, in adult male zebra finches. $\boldsymbol{a}$, The packing density of Hu-positive neurons in HVC significantly increases between $90 \mathrm{~d}$ and 9 years of age in adult male zebra finches $(p=0.0002) \cdot \boldsymbol{b}$, In the same group of birds, there is no significant change in the packing density of Hu-positive neurons with age in an area of NC just ventral to HVC (see Fig. 1c). Each data point represents the counts from either left or right HVC within one bird. There was no systematic difference in neuron density between the left and right hemispheres in either HVC or NC densities.

with age (linear regression: $R^{2}=0.40, p=0.003$ ). Although the significance of nucleus shrinkage is unclear, evidence from rat motor neurons suggests nuclear and cytoplasmic shrinkage are correlated (Geinismann et al., 1971), and so it is likely that the HVC-RA neurons also show a reduction in soma size with age. Thus, the increase in the total number of HVC-RA neurons appears to be accompanied by both a shrinking of the neurons and an increase in their packing density.

New neurons are added to many parts of the telencephalon in adult songbirds, not just HVC (Nottebohm, 1985; Vellema et al., 2010). We tested a second brain area to determine whether net neuron addition was a general feature of the adult zebra finch brain or was more specific to HVC. Using the same slides that had been stained for Hu and used to count HVC neurons, we counted $\mathrm{Hu}$-positive neurons in an area of dorsal NC, just below HVC (Fig. 1c). This area receives auditory inputs from field $\mathrm{L}$ (Vates et al., 1996) and recruits new neurons in adulthood (Nottebohm, 1985; Vellema et al., 2010). Because the entire area of NC was not contained within our sections, we could not calculate the volume of NC. Thus, counts of $\mathrm{Hu}$-positive neurons were only calculated as neuron density per cubic millimeters. In contrast to HVC, we found no change in the packing density of $\mathrm{Hu}$-positive neurons in this region of NC between $90 \mathrm{~d}$ and 9 years of age (Fig. $3 b$; linear regression for neuron density: $R^{2}=0.08, p=0.30$ ). There was a slight but nonsignificant trend toward a density increase with age but the trend completely disappeared when the outlying 9-year-old bird was removed from the dataset. Therefore, the increase in neuron density we report for HVC in this group of birds is not a general feature of adult telencephalon. At $90 \mathrm{~d}$ of age, HVC and the adjacent $\mathrm{NC}$ contain neurons packed at equal density, $\sim 250,000$ neurons per $\mathrm{mm}^{3}$. Adult-born neurons are recruited into both brain areas across adulthood but only in HVC is there a net gain in packing density. In the NC region, neuronal recruitment is perhaps accompanied by neuron death, as in the canary HVC (Kirn and Nottebohm, 1993; Kirn et al., 1994,) resulting in numerical replacement and a constant neuron density.

\section{Modeling the net addition of HVC-RA neurons in adulthood}

There are two possible ways to achieve a net addition of neurons: either all the neurons added to HVC survive and therefore the rate of population growth equals the rate of new neuron incorporation, or, if some neuronal replacement occurs, then the rate of new neuron incorporation is greater than that of neuron death. To explore these two possible scenarios for the zebra finch HVC, we used previously published data to predict how the HVC-RA population would grow if all new neurons survived indefinitely. Wang et al. (2002) published data showing that the number of new HVC-RA neurons recruited to the adult HVC decreases as zebra finches gets older. They injected birds ranging in age from $90 \mathrm{~d}$ to 3 years with ${ }^{3} \mathrm{H}$-thymidine, waited 4 months, injected a retrograde tracer into nucleus RA to label the HVC-RA neurons, and then counted the number of ${ }^{3} \mathrm{H}$-positive HVC-RA neurons surviving in HVC. They found that the number of labeled neurons declined as the age of the bird at the time of ${ }^{3} \mathrm{H}$-thymidine injection increased and that this decline was not linear with respect to age (Wang et al., 2002). These data do not distinguish between an age-related decline in the production of new neurons and an age-related decline in the survival of new neurons in HVC; they just tell us that the number of neurons that was still present in HVC after 4 months declined with age. We assumed that every HVC-RA neuron that survived in HVC for 4 months would survive indefinitely, and then used the Wang et al. (2002) data to predict how the total HVC-RA population would change with age if the assumption were correct. We used nonlinear regression to derive an exponential decay rate constant for the change in 4 month survival with age and integrated the rate equation to find the total accumulation of new HVC-RA neurons in a given time period (see Materials and Methods for the equations). Since the study from Wang et al. (2002) used adult birds aged $90 \mathrm{~d}$ or older, the integration was performed from day 90 onwards as the dynamics of neuron addition to HVC could be very different in the juvenile period.

Using this integral equation and an experimentally derived value for the average number of HVC-RA neurons in a 90-d-old bird, we generated a set of predicted values for the total number of HVC-RA neurons expected at a given age if the assumption of complete neuronal survival were correct. Using an average value for HVC volume, these predicted values were converted to HVC-RA neuron densities and compared with our observed data for HVC-RA neuron density between $90 \mathrm{~d}$ and 11 years of age (Fig. 4). The integral equation provides quite a good approximation of the observed data and gives an $R^{2}$ value of 0.65 , which is impressive given that it was derived from experimental data produced by a different laboratory using a different set of birds. The asymptote of the predicted values is higher than the true asymptote, which could be the result of some neuron death occurring in older birds but also could just reflect differences in the methods used to obtain the two datasets. For example, Wang et al. (2002) used fluorogold as a retrograde tracer and we used CTB, and the 


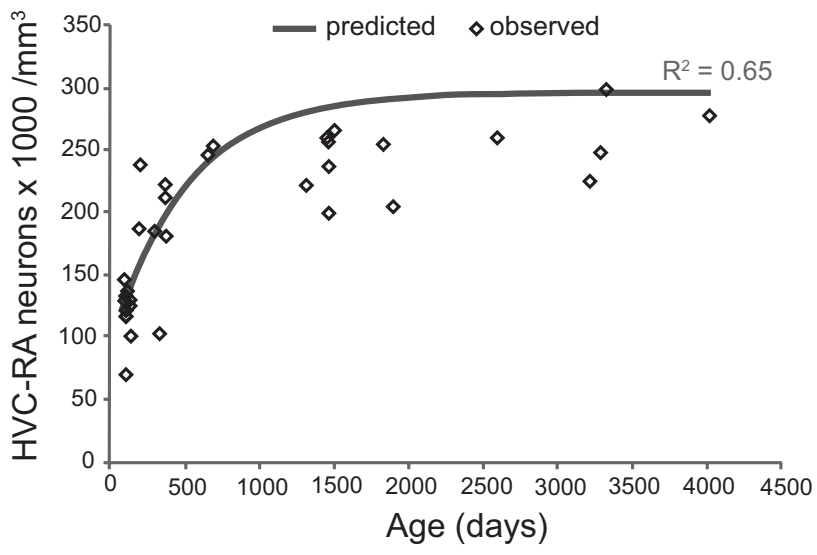

Figure 4. A model that assumes complete survival of new HVC-RA neurons approximates the age-related changes we see in the HVC-RA neuron density with age. Using a rate equation derived from data in Wang et al. (2002) showing how HVC neuron recruitment changes with age in adult zebra finches, a set of predicted values for the total number of HVC-RA neurons that would be present in birds of different ages was generated. These predicted values assumed that every neuron that was recruited to HVC and survived for 4 months in Wang et al. (2002) would go on to survive indefinitely, and that neuron addition was accompanied by no loss of the preexisting HVC-neuron cohort. Predicted values for total HVC-RA neuron number were converted to HVC-RA neuron density using a canonical volume for HVC. The predicted values (gray line) that assume complete survival of new HVC-RA neurons were compared with the observed values for HVC-RA density and age (diamonds). The model comes close to the real change in HVC-RA neuron density with age that we have measured $\left(R^{2}=0.65\right)$.

labeling efficiency of these two tracers may differ. Alternatively, the different asymptotes could be due to a baseline difference in the rate of neuronal recruitment to HVC, or a difference in the mean number of HVC-RA neurons present at day 90, between the two cohorts of birds. We cannot distinguish between these possibilities. However, even if our assumption of complete neuron survival is incorrect and there is some death of the HVC-RA neurons, the relative similarity between the predicted and observed curves suggests that most new neurons added to HVC after day 90 survive for the long term and that neurogenesis in the adult zebra finch HVC results, predominantly, in the net addition of neurons. This is in stark contrast to observations made in the canary where new HVC neurons are often short-lived and numerically replace older neurons that have died. While in the adult zebra finch HVC neurogenesis results in net growth in the number of HVC-RA neurons, in the canary it appears to just maintain a fixed HVC-RA population size.

\section{Experiment 2: Is there also neuronal replacement in adult zebra finch HVC?}

Our modeling suggested that some neuronal replacement could accompany the addition of new neurons to the adult zebra finch HVC. To test this theory, we retrogradely labeled the HVC-RA neurons in young adults and followed their survival over the next 9 months. If some HVC-RA neurons were being numerically replaced, we would expect to see a reduction in the number of labeled HVC-RA neurons over this period, as the labeled neurons were replaced by new unlabeled ones. Latex microspheres were used as the retrograde tracer because they had been used to show loss of HVC-RA neurons over a similar time period in canaries (Kirn and Nottebohm, 1993) and because microsphere labeling persists for at least a year in vivo (Katz et al., 1984). Birds were injected bilaterally with red fluorescent latex microspheres into $\mathrm{RA}$ at $\sim 95 \mathrm{~d}$ of age, and the number of retrogradely labeled HVC-RA neurons was counted after $1,3,6$, or 9 months. There

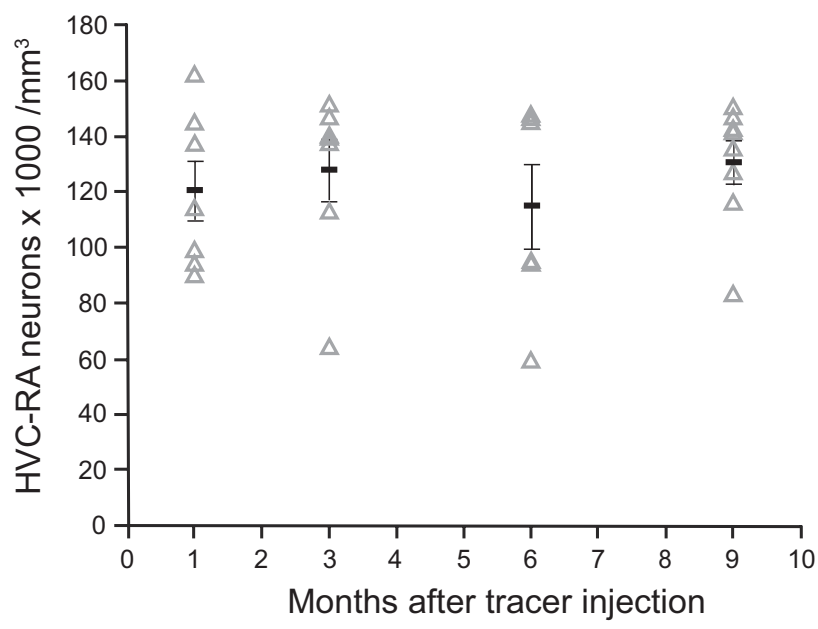

Figure 5. The number of HVC-RA neurons retrogradely labeled at $95 \mathrm{~d}$ of age does not change during the following 9 months. Counts of HVC-RA neurons retrogradely labeled with latex microspheres injected into RA at 95 d of age are similar after 1, 3, 6, and 9 months survival time. Each data point represents the mean between left and right HVC within one bird. Group means are represented as black bars. Error bars represent SEM.

was no change in the total number or density of retrogradely labeled neurons across the four survival groups (Fig. 5; for density, one-way ANOVA: $F_{(3,27)}=0.42, p=0.74$; for total number, one-way ANOVA: $\left.F_{(3,27)}=0.91, p=0.45\right)$, suggesting no appreciable loss of HVC-RA neurons had occurred over the 9 months. In a similar experiment in adult canaries, in which the survival time straddled spring, summer, and fall, there was almost a 50\% loss of retrogradely labeled HVC-RA neurons over a 7 month period (Kirn and Nottebohm, 1993).

It is possible that the HVC-RA neurons retrogradely labeled in a 95-d-old zebra finch, and therefore those born earlier during the juvenile song learning period behave differently from HVC-RA neurons born in adulthood. HVC-RA neurons born after sexual maturity could serve a role other than encoding the motor pattern of song and thus could be part of a more transient and replaceable neuron population. To look for neuronal replacement of adult-born neurons, the males in Experiment 2 were also given injections of BrdU on posthatching days 92, 93, and 94 (just before the microsphere injection surgery on day 95) to birthdate label new neurons born at this time. The number of BrdU-positive neurons present in HVC was counted 1, 3, 6, or 9 months later. In addition, a fifth group of birds was injected with the same protocol of BrdU and killed 4 years later. Six days before they were killed, birds in this fifth group received injections of a retrograde tracer (green CTB) into RA to determine what proportion of any surviving BrdU-positive neurons were also HVC-RA neurons.

We were surprised to find BrdU-positive neurons ( $\mathrm{Hu}-$ positive) in the HVCs of the 4-year survival birds. These birds received BrdU injections at 92-94 d of age and so the labeled neurons were 3.75 years old. Approximately $50 \%$ of these BrdUpositive neurons were also retrogradely labeled with CTB from injections made into RA $6 \mathrm{~d}$ before the birds were killed (Fig. 6a). This is the first time that adult-born neurons have been shown to persist for many years in the HVC of a songbird. To estimate how many of the neurons born between 92 and $94 \mathrm{~d}$ of age had survived for 4 years, we counted all the $\mathrm{BrdU} / \mathrm{Hu}$ double-positive cells within HVC and compared the counts to those made in the $1,3,6$, and 9 month survival groups. There was no difference in the total number or density of BrdU-positive neurons in HVC 
between any of the survival times (Fig. $6 b$; for density, one-way ANOVA: $F_{(4,41)}=$ $1.35, p=0.27$; for total number, one-way ANOVA: $\left.F_{(4,41)}=1.89, p=0.13\right)$. Thus, there was no significant loss of adult-born neurons in these birds between the time of the earliest count, at 1 month survival, and 4 years of age. This outcome is compatible with the observation that adultborn neurons accumulate in the HVC of adult zebra finches, a process of net gain with no evidence of loss.

There is a potential caveat in the interpretation of these survival data. The method of using retrograde tracers to determine the age of a neuron is confounded by the possibility that tracer at the injection site remains available for transport back to HVC after the original injection. If it did, new neurons picking up tracer weeks or even months after the injection surgery could mask any loss of some of the original retrogradely labeled cohort. We found evidence for this possibility: some of the BrdU-positive neurons born immediately before the injection of latex microspheres to RA were seen to have transported microspheres back to their cell bodies in HVC, even though experiments in canaries have shown that it takes 2 weeks or more for the axons of adult-born neurons to innervate RA (Kirn et al., 1999). Thus, it seems that microspheres present in RA were taken up by some neurons connecting after the initial injection surgery. This caveat did not pose a problem for the counts of CTB-positive HVC-RA neurons in Experiment 1, because survival time was fixed in this experiment, with all birds killed $6 \mathrm{~d}$ after CTB injection. However, in Experiment 2, survival time was the variable studied. In this case, uptake of tracer by neurons born weeks or months after tracer injection could have concealed a loss of members of the original cohort of microsphere-labeled HVC-RA neurons.

We tried to estimate the extent to which HVC-RA neuron loss could have been masked by new neurons becoming labeled after the tracer injection. We counted the number of BrdU/Hu/microsphere triple-positive neurons in each survival group and used these counts to calculate the percentage of all BrdU/Hu neurons that were also positive for microspheres. This percentage represented the proportion of new neurons recruited to HVC after the tracer injection that took up microspheres from the deposit in RA. The percentage was variable between birds but the means were not significantly different across the four survival times (Fig. $7 a$; one-way ANOVA: $\left.F_{(3,29)}=1.84, p=0.14\right)$. Taking an average of all four groups, $7.5 \%$ of all the BrdU-labeled neurons were also labeled with microspheres from RA. Thus, 0.075 provided a rough indication of the probability that a new neuron recruited to HVC after the injection of microspheres would take up tracer to become labeled and therefore get included in our counts of retrogradely labeled HVC-RA neurons.

Next, we estimated the number of new neurons that would have been recruited to HVC during the time between the microsphere injection and the time of death for each survival group using the integrated rate equation derived from the data in Wang et al. (2002), as described above. The rate equation, which describes how neuronal recruitment to HVC changes with age, was integrated between day 95 and the day on which each group was killed, to estimate the total number of neurons that would have been added to HVC after the microsphere injection for each
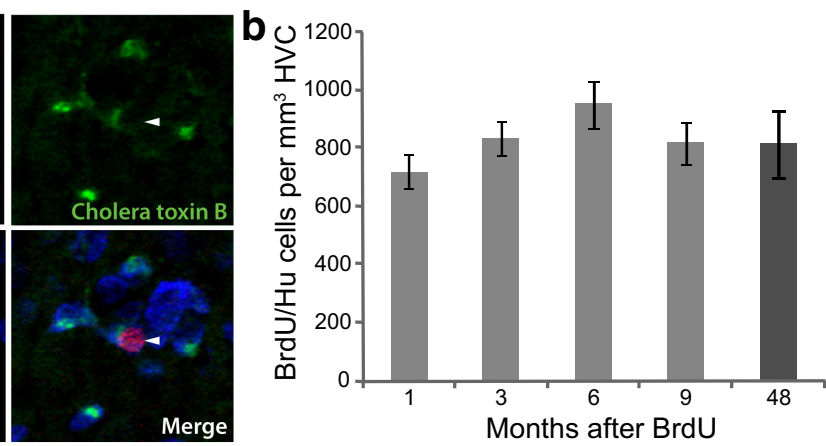
Figure 6. Neurons born at 92-94 d of age are still present in HVC 4 years later. a, Many BrdU/Hu double-positive neurons were
present in HVC of birds killed 4 years after BrdU injections at 92-94 d of age. A significant number of them could also be retrogradely neuron. $\boldsymbol{b}$, Counts of BrdU/Hu double-positive neurons in HVC at various survival times after BrdU injections at 92-94 d of age revealed that there was no loss of labeled cells between 1 month and 4 years. Error bars represent SEM.

group. These estimates were then multiplied by 0.075 to estimate the total number of "extra" microsphere-positive neurons that would have been included in the total counts of retrogradely labeled HVC-RA neurons. Subtracting these final estimates from the raw counts of microsphere-positive HVC-RA neurons provided new total HVC-RA numbers that were more likely to represent the true number of the originally labeled HVC-RA cohort present in each survival group. Figure $7 b$ shows the corrected counts of microsphere-positive HVC-RA neurons after removing the potential masking effect of extra newly labeled neurons. There is still no significant difference between survival groups (one-way ANOVA: $F_{(3,27)}=0.69, p=0.57$ ). We appreciate that because these estimates are based on experimentally derived data from a different laboratory using different birds (Wang et al., 2002), they may not be very accurate at representing the actual number of newly labeled neurons that could have been counted in our experimental birds. However, because the difference between the raw and corrected HVC-RA neuron counts is so small, even if these estimates are not entirely accurate, we would argue that any masking effect of retrogradely labeled neurons added after the original microsphere injection is negligible compared with the levels of the net addition we describe in Experiment 1. Thus, even accounting for the potential limitations of the experimental method, we still find no appreciable loss of the HVC-RA neurons present in 90-d-old male zebra finches over the next 9 months of life.

There could have been some concern, too, about how to interpret the counts of BrdU-positive neurons at different survival times after injection of BrdU. The simplest assumption is that BrdU-positive neurons counted in HVC were born during the $3 \mathrm{~d}$ of BrdU injections. However, in reality, it is possible that the neuronal progenitor cells dividing in the $\mathrm{VZ}$ at the time of BrdU exposure retain enough BrdU in their DNA to continue to produce BrdU-positive daughter neurons in subsequent divisions. Thus, the same confounding factor described for microspherepositive neurons is possible with the counts of BrdU-positive neurons in Experiment 2: a loss of the original BrdU-positive cohort could have been masked by the influx of new BrdUpositive neurons adding to HVC after the time of BrdU injections. We did not have enough information to be able to estimate how much of an effect these extra BrdU-positive neurons could have on the total counts at each survival time. These calculations would have required information on how often each progenitor cell divides to produce new neurons, whether this rate changes 

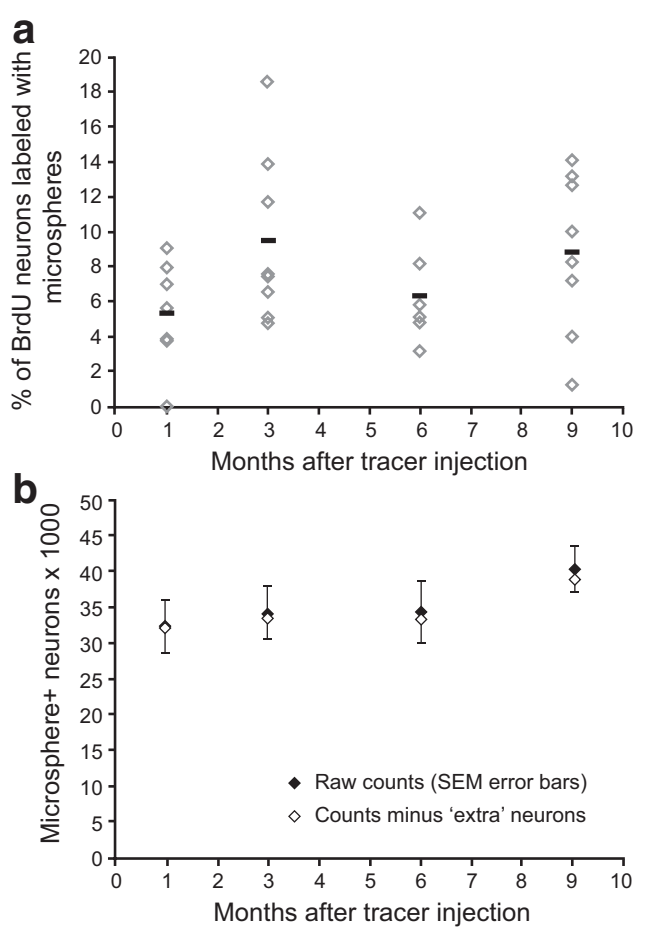

Figure 7. Microspheres can be transported from RA after the initial injection but the number of additionally labeled neurons does not dramatically alter the final HVC-RA neuron counts. $\boldsymbol{a}$, At all time points after microsphere injection, some of the BrdU-positive neurons were also labeled with microspheres from RA, even though they would not have been connected to RA at the time of microsphere injection. The percentage of BrdU-positive neurons labeled with microspheres was not significantly different between survival times $(p=0.14)$. Across all survival times, on average, $7.5 \%$ of the BrdU-positive neurons were retrogradely labeled with microspheres. Each data point represents the mean between left and right HVC of one bird. Group means are represented as black bars. Error bars represent SEM. $\boldsymbol{b}$, The total number of microsphere-positive HVC-RA neurons present at each survival time (black diamonds) was corrected for potential extra labeled neurons that were recruited after the microsphere injection but still took up microspheres from a deposit in RA. Extra labeled neurons were estimated for each survival time using published data on the rate of neuronal recruitment to HVC (Wang et al., 2002) and a mean probability that $7.5 \%$ of them will take up microspheres. Even correcting for these potential extra labeled neurons (white diamonds), there is no significant difference between the total number of microsphere-positive HVC-RA neurons present at 1, 3, 6, or 9 months after microsphere injection at $95 \mathrm{~d}$ of age $(p=0.57)$.

with age and also how many divisions can occur before the BrdU is diluted enough that a daughter neuron no longer appears BrdU-positive.

We used an indirect way of assessing to what extent addition of new BrdU-labeled neurons could have masked the loss of BrdU-labeled neurons born between 92 and $94 \mathrm{~d}$ of age. There is evidence that progenitor cells that supply HVC with new neurons in adulthood are located in the VZ above HVC (Goldman and Nottebohm, 1983; Scott and Lois, 2007). We counted the number of BrdU-positive cells present in the VZ above HVC (Fig. 8a) at 1, 3, 6, 9, and 48 months after the BrdU injections to determine whether the progenitors had continued to divide after the initial labeled division. Our counts revealed a rapid decrease in the number of labeled VZ cells with increasing survival time (Fig. 8b), which is in agreement with an earlier zebra finch study that reported a precipitous drop in the number of labeled VZ cells in the first month after exposure to the birth-date marker (AlvarezBuylla et al., 1988). The decline in BrdU-positive VZ cells we observed suggests that either some of the labeled VZ cells died, or more likely, they continued to divide until the BrdU was too diluted to meet our labeling criteria for a BrdU-positive cell.
Thus, it is possible that a continued influx of new BrdU-positive neurons born after the $3 \mathrm{~d}$ of BrdU injections in Experiment 2 birds concealed the loss of some members of the originally labeled neuron cohort, particularly during the shorter survivals of 1-3 months. But, because the number of labeled VZ cells dropped off so rapidly and because we are dealing with survival times of up to 4 years, it is unlikely that an influx of newly labeled BrdU neurons would have significantly contributed to the longer term total number of BrdU-positive neurons counted in HVC. We suggest that the more parsimonious interpretation of the data is that losses that may occur between 1 and 3 months are modest and that there is no significant loss of the originally labeled cohort between 3 months and 4 years.

\section{What kinds of HVC neurons are produced in adulthood?}

The evidence that HVC-RA neurons but not HVC-X neurons are produced in adulthood is robust in both canaries and zebra finches: neurons that are birthdate-labeled in adults can be retrogradely labeled with tracers from RA but not Area X (Alvarez-Buylla et al., 1990; Kirn et al., 1991, 1999; Kirn and Nottebohm, 1993; Scharff et al., 2000; Scotto-Lomassese et al., 2007). However, in these labeling experiments there is always a proportion of new birthdate-labeled neurons that are not retrogradely labeled with tracer from RA, suggesting that either there is a subset of HVC-RA neurons that, for whatever reason, does not carry the label retrogradely from RA to the soma in HVC, or that not all the neurons recruited to the adult HVC project to RA. In the canary, the number of new neurons that can be retrogradely labeled with tracer from RA increases from $\sim 50 \%$ at 1 month after injection of the birthdate marker to $\sim 80 \%$ after 8 months (Alvarez-Buylla et al., 1990; Kirn et al., 1991), suggesting that it takes some time before some of the new HVC-RA neurons are competent to transport the tracer back to HVC. The same phenomenon could also be true in the zebra finch HVC. One month after BrdU injections in adult zebra finches, $42 \%$ of the BrdU-positive neurons in HVC can be retrogradely labeled from RA (ScottoLomassese et al., 2007), but perhaps if a long enough time were allowed between birthdate labeling and injection of the retrograde tracer, all the new neurons would be labeled. This is not what we observed; in the 4-year survival birds from Experiment 2, we found that only $50 \%$ (range $34-64 \%$ ) of the new neurons were labeled with CTB from RA, even after allowing 4 years for a connection with RA to be established. If an adult-born neuron cannot be retrogradely labeled from RA after 4 years, it is unlikely that it ever will.

In addition to the two kinds of projection neuron, HVC also contains a substantial proportion of interneurons whose axonal arbors are completely contained within HVC (Nixdorf et al., 1989; Mooney, 2000). These interneurons have been shown to be produced postnatally, in juveniles between 28 and $48 \mathrm{~d}$ of age, but in far fewer numbers than new HVC-RA neurons in birds of this age (Scott and Lois, 2007). In contrast, attempts to label adultborn neurons with interneuron markers (GABA, parvalbumin, calretinin, or calbindin) have been unsuccessful (ScottoLomassese et al., 2007), suggesting that the production of interneurons drops off after sexual maturity. One potential caveat of the labeling study conducted by Scotto-Lomassese et al. (2007) was that killing the birds 1 month after BrdU injections could have been insufficient for the new neurons to fully mature into interneurons and begin expressing the interneuron marker proteins. If this caveat were valid, we would expect that 4 years would be sufficient for full interneuron maturation and that we would be able to see colocalization of BrdU with interneuron markers in 
the HVCs of our 4-year survival birds, if indeed interneurons are produced in the adult brain. We stained their HVC tissue sections with a mixture of antibodies against parvalbumin, calretinin, and calbindin, three calcium-binding proteins previously shown to label HVC interneurons (Wild et al., 2005). We observed many positively stained interneurons in HVC but found no colocalization between the interneuron markers and BrdU in any of the six birds examined (Fig. 9). Thus, even after allowing 4 years for the adult-born neurons to mature, we found no evidence that any of them became local interneurons in the HVC of adult zebra finches. At this stage, the identity of neurons recruited to HVC that cannot be retrogradely labeled from RA still remains a mystery.

Experiment 3: Age and social environment affect HVC neuron recruitment but not neuron survival between 1 and 3 months Zebra finches are a social species that typically live in large colonies in the wild. We wondered whether the manner in which we housed our laboratory birds, usually one per cage, might have influenced the number of new neurons recruited and their subsequent survival. We also wondered whether the adult age at which new neurons were born and labeled affected their subsequent survival. Might new neurons born later in adulthood show reduced survival than new neurons born earlier in adult life? To explore the effects of age and social environment on the survival of adult-born neurons in HVC, we counted the number of BrdUpositive neurons in $\mathrm{HVC}$ at 1 and 3 months after BrdU injections in two additional groups of male zebra finches. Birds in one of these groups (young, socially housed) were of the same age as those in our original Experiment 2 (92-94 d), but in this case were placed in a social aviary setting immediately after BrdU injections until they were killed. Birds in the other group were 2 years old and housed in single cages after BrdU treatment (old, singly housed). Counts of BrdU/Hu double-positive HVC neurons in these two additional groups were compared with those made in the Experiment 2 birds (hereafter called young, singly housed). In the old, singly housed birds, the number of $\mathrm{BrdU} / \mathrm{Hu}$ doublepositive HVC neurons at 1 month was a quarter of the number recruited in young, singly housed birds (Fig. 10a; Mean \pm SEM: $175 \pm 32$ neurons per $\mathrm{mm}^{3}$ for old birds, $735 \pm 60$ neurons per $\mathrm{mm}^{3}$ for young birds), an age-related decline similar to that observed by Wang et al. (2002). In the young, socially housed birds, the number of neurons recruited in the first month was twice that of the young, singly housed birds (Fig. 10a; Mean \pm SEM: $1436 \pm$ 138 neurons per $\mathrm{mm}^{3}$ for socially housed, $735 \pm 60$ neurons per $\mathrm{mm}^{3}$ for singly housed), replicating the effect found by Lipkind et al. (2002).

Next, we compared the number of BrdU-positive neurons present in HVC 3 months after BrdU injections in the old, singly housed; young, singly housed; and young, socially housed birds. There was no significant difference in the number of new HVC neurons between 1 and 3 months in any of the three experimental conditions and no additive effect between group and survival time (Fig. 10a; unweighted-means two-way ANOVA: effect of group, $F_{(2,46)}=12.17, p<0.0001$; effect of survival time, $F_{(1,46)}=$ $3.27, p=0.078$; interaction effect, $\left.F_{(2,46)}=0.21, p=0.81\right)$. Thus,

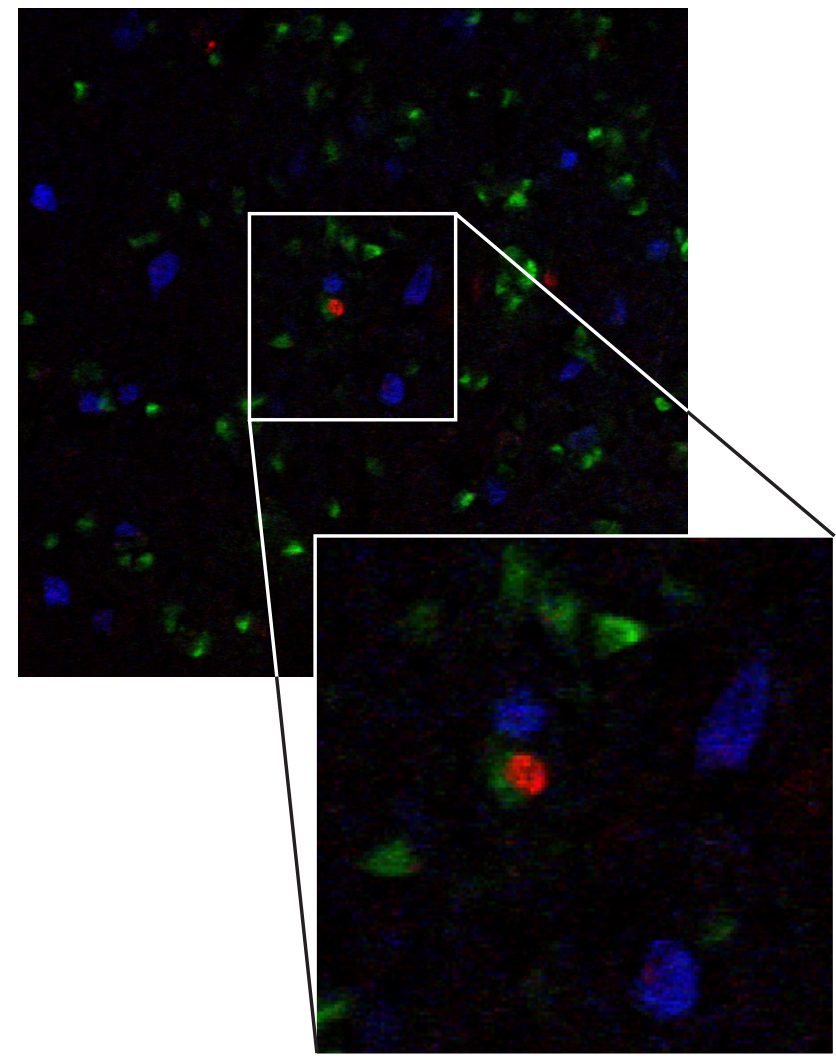

Figure 9. Four-year-old adult-born neurons in HVC do not express interneuron markers. A mixture of antibodies against the calcium-binding proteins calbindin, calretinin, and parvalbumin stains many interneurons in HVC (blue) but there is no colocalization between the interneuron markers and BrdU (red) in birds that had received BrdU injections at $90 \mathrm{~d}$ of age and were killed 4 years later. HVC-RA neurons are labeled with CTB retrograde tracer injected into RA before killing (green). The enlarged panel shows the BrdU-positive nucleus of a retrogradely labeled HVC-RA neuron surrounded by three interneurons positive for the mix of calcium binding proteins.

while both age and social environment influenced the overall recruitment of new neurons into HVC during the first month after BrdU exposure, neither variable affected survival of the new cells during the two subsequent months. Rather, there was in all three conditions a nonsignificant trend toward more new neurons at 3 months than at 1 month, possibly because during that 


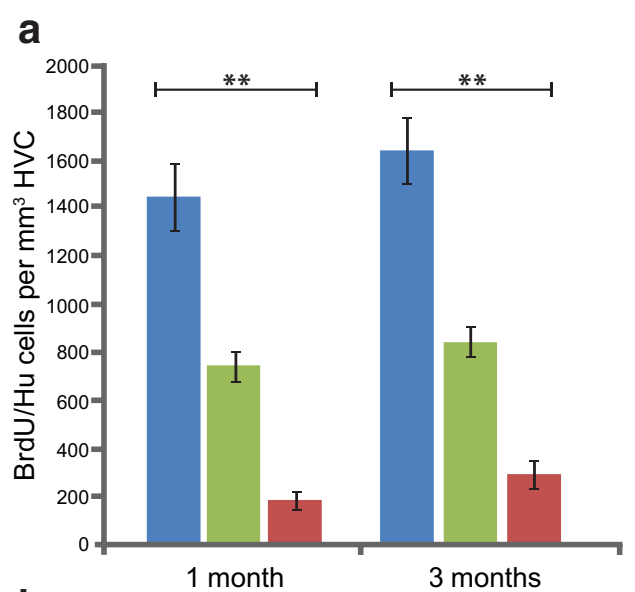

b

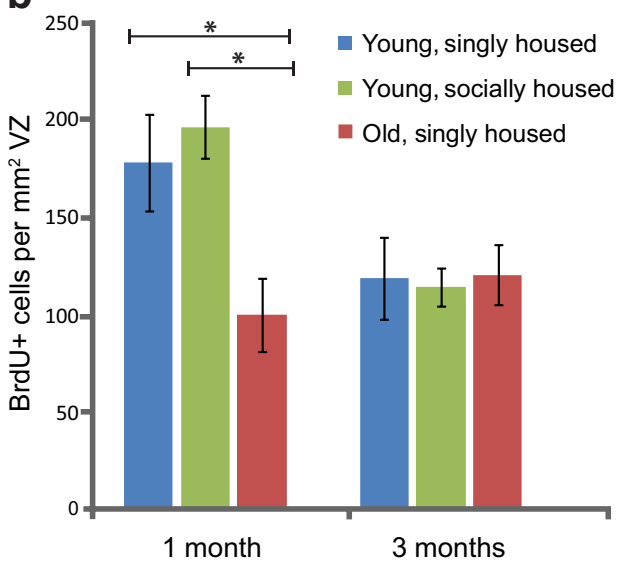

Figure 10. Age and social environment affect the recruitment of new neurons to adult HVC but not the survival of new neurons between 1 and 3 months. $\boldsymbol{a}, 0$ ne month after BrdU injection, the young, socially housed adults had recruited twice as many BrdU-positive neurons to HVC and the old, singly housed birds had recruited four times fewer BrdU-positive neurons to HVC than the young, singly housed birds. There was no loss of BrdU-positive neurons in HVC between 1 and 3 month survival in any of the three groups. $\boldsymbol{b}$, There was no difference in the number of BrdU-positive VZ cells between the young, socially housed and the young, singly housed groups at either 1 or 3 months survival times, and both young groups showed a significant reduction in the number of BrdU-positive neurons between 1 and 3 months. Old, singly housed birds had half the number of BrdU-positive VZ cells 1 months after BrdU injections than the number in either young group but they did not show any loss in the number of labeled VZ cells between 1 and 3 months, suggesting no further division of the progenitors occurred. ${ }^{*} p<0.01{ }^{* *} p<0.0001$.

interval some labeled VZ neuronal progenitors continued to divide and produce labeled neurons.

We did not know whether the effects of age and social environment on HVC neuronal recruitment during the first month after BrdU exposure were mediated by changes in the production, migration, or survival of new neurons. To test for the contribution of increased neuron production, we compared the number of BrdU-positive VZ cells above HVC 1 month after BrdU injection in birds from the old, singly housed; young, singly housed; and young, socially housed groups. There were 50\% fewer labeled progenitors in the old birds compared with both young groups but there was no difference between the singly and socially housed young birds (Fig. 10b; Mean \pm SEM: young, singly housed $=196 \pm 16$ cells; young, socially housed $=178 \pm 25$ cells; old, singly housed $=100 \pm 18$; two-way ANOVA followed by Tukey HSD post hoc analysis: $p<0.01$ for old birds compared with either of the young groups at 1 month, all other comparisons not significant). After 3 months, all groups had the same number of BrdU-positive progenitor cells (Fig. 10b; Mean \pm SEM: young, singly housed $=114 \pm 10$ cells; young, socially housed $=119 \pm$ 21 cells; old, singly housed $=120 \pm 15$ ). This came about because both young groups showed a decrease in the number of labeled VZ cells between 1 and 3 month survivals while there was no difference between the two survival times in the older adults.

Together, the above results suggest that aging reduced the number of actively dividing progenitors in the VZ above HVC and also reduced the frequency with which each cell divided because there was no loss in the number of labeled VZ cells between 1 and 3 month survivals in the 2-year birds. In contrast, a more complex social environment did not appear to affect cell division in the VZ above HVC, but it induced a doubling in the number of new neurons that were present in HVC at the 1 month survival. This result suggests that while the same number of new neurons entered HVC in the socially and singly housed young adults, social complexity had already favored a greater survival of these cells by the time they were counted 1 month after BrdU injection. From work in the canary, we know that adult-born neurons take between 1 and 2 weeks to migrate into HVC and assume an adult, sedentary phenotype, but then between 2 weeks and 1 month after birth there is substantial culling of the neurons, with only half of the original cohort surviving to 1 month (Kirn et al., 1999; Alvarez-Borda et al., 2004). If neuronal recruitment into the HVC of adult male zebra finches follows a similar timetable, then a socially complex setting appears to rescue many of the neurons that normally die within the first month.

To test this latter interpretation, we looked for a correlation between the number of labeled VZ cells present 1 month after $\mathrm{BrdU}$ injections and the number of $\mathrm{BrdU} / \mathrm{Hu}$ neurons surviving in HVC after the same time period. If division of progenitors in the $\mathrm{VZ}$ is the limiting factor in the recruitment of new neurons to HVC, then there should be a positive correlation between the number of labeled VZ cells and the number of labeled neurons among individual birds. However, if many of the new neurons are culled before fully integrating into HVC, then the correlation between these two factors will be weak. We found a positive correlation between BrdU-positive HVC neurons and BrdUpositive VZ cells but only in the group of young, socially housed birds (Fig. 11; young, socially housed: $R^{2}=0.68, p=0.02$; young, singly housed: $R^{2}=0.043, p=0.59$; old, singly housed: $R^{2}=$ $0.015, p=0.81)$. In singly housed birds, both young and old adults, there is no relationship between the amount of cell division in the VZ above HVC and the number of new neuron present in HVC after 1 month; a ceiling effect occurs, presumably because many of the new neurons are killed within the first month. In the socially housed young adults, there is an increase in the survival of new HVC neurons during the first month after their birth (Fig. 10a) and, as a result, the number of new neurons present in HVC at 1 month becomes dependent on the extent of cell division in the overlaying VZ; the ceiling effect is removed. This result bears on our interpretation of the outcome from Experiment 2. The absolute numbers counted after survivals of 1, 3, 6 , and 9 months and at 4 years might be an underestimate of the total number that would have survived had the social setting been more complex.

\section{Discussion}

The high vocal center (HVC) of the avian song system is a convenient place in which to study how adult neurogenesis affects brain function because (1) new neurons are recruited into adult HVC; (2) HVC participates in the narrowly defined task of vocal communication; and (3) HVC is found in all songbirds (Wild, 1997), thus providing good material for comparative studies. 


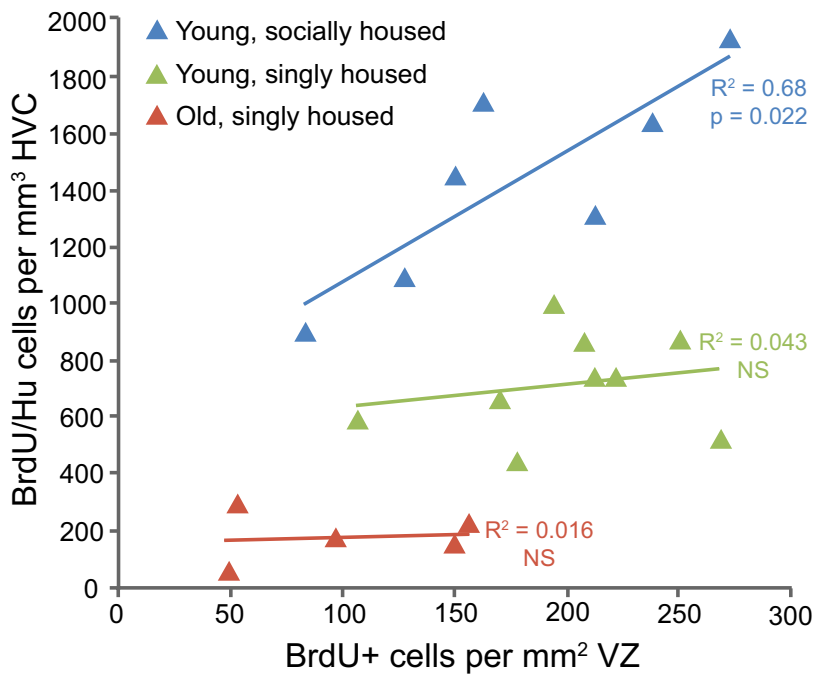

Figure 11. The number of new neurons in HVC is only correlated with the number of dividing progenitors in the young, socially housed birds. BrdU-positive neurons in HVC were significantly correlated with the number of BrdU-positive cells in the ventricular zone in the young, aviaryhoused birds $\left(R^{2}=0.68, p=0.022\right)$ but not in the young, singly housed or old, singly housed groups.

In canaries and song sparrows, HVC neurons are culled at the end of the breeding season, leading to a smaller HVC with fewer neurons (Nottebohm, 1981; Kirn et al., 1994; Smith et al., 1997). In both species new neurons are recruited into HVC year-round, but their addition is highest in the fall (Kirn et al., 1994; Tramontin and Brenowitz, 1999). Fall shrinkage in HVC volume has also been reported in adults from other seasonal songbirds (Arai et al., 1989; Bernard and Ball, 1995; Brenowitz et al., 1991, 1998; Smith et al., 1995), but direct evidence for neuronal replacement in these species is still missing. In all cases examined, adult song is less stereotyped in the fall (Brenowitz, 2004), but we do not know whether song instability and neuronal turnover are causally related.

Zebra finches, too, show neurogenesis in adult HVC, but the context and consequences differ from those described in canaries and song sparrows. Zebra finch song does not show seasonal instability and it changes very little after sexual maturity (Immelmann, 1969). Here, we show that recruitment of new HVC neurons in adult zebra finches is not part of a process of turnover but one of net addition. We infer that culling of new HVC neurons can occur within the first $30 \mathrm{~d}$ after neuron birth because housing birds in a social environment rescues many new neurons that otherwise would not survive. However, we found no evidence of neuron loss thereafter; adult-born HVC neurons that survived the first month went on to survive for up to 4 years. Moreover, using the same techniques that revealed substantial neuron loss in adult canaries (Kirn and Nottebohm, 1993; Alvarez-Borda et al., 2004) showed a doubling of HVC-RA neuron numbers over the lifespan of adult zebra finches. HVC did not grow to accommodate the extra neurons, instead they became more densely packed with age. We observed no similar increase in neuron packing density in an area of NC just below HVC, which is congruent with a previous report that neurons recruited to the adult zebra finch NC are transient (Barnea et al., 2006).

What function could increasing numbers of HVC-RA neurons serve in the adult zebra finch? Electrophysiological recordings in awake, singing birds suggest HVC-RA neurons encode the motor pattern for song (Hahnloser et al., 2002). There is some evidence that the song pattern (Pytte et al., 2007) and the syllable acoustic structure (Kao and Brainard, 2006) of zebra finch song become more stereotyped with age and that this may give older males a mating advantage (Woolley and Doupe, 2008). Maybe the addition of HVC-RA neurons in adulthood promotes song stereotypy. Motor output from RA is initially dominated in juveniles by input from the lateral magnocellular nucleus of the anterior nidopallium (IMAN) (Herrmann and Arnold, 1991). LMAN neurons fire in a variable manner when the bird sings and are responsible for the variable song of juveniles (Hessler and Doupe, 1999; Olveczky et al., 2005; Aronov et al., 2008). This variability is necessary for song learning (Bottjer et al., 1984; Scharff and Nottebohm, 1991). As song learning progresses, control of RA shifts toward the stereotyped firing pattern of the HVC-RA neurons and song becomes gradually more stable (Herrmann and Arnold, 1991; Aronov et al., 2008). Since IMAN-RA neurons continue to generate some variability in adult song (Kao and Brainard, 2006), it is conceivable that more HVC-RA neurons in adulthood further dilute the influence from IMAN and result in an age-related increase in song stereotypy. This, however, would be a roundabout way of achieving something that could be done by reducing IMAN activity. LMAN, we know, is not necessary for the production of adult song (Bottjer et al., 1984; Scharff and Nottebohm, 1991).

Perhaps adult-born neurons are involved in song perception, not production. Many HVC neurons respond to sound (Katz and Gurney, 1981). These responses are selective to conspecific song (Margoliash, 1983) and particularly to playbacks of the bird's own song (Margoliash, 1986), a feature that is shown by both HVC-RA and HVC-X neurons (Mooney, 2000). Experiments using electrophysiology and immediate-early gene expression in adult zebra finches show that not all HVC-RA neurons are active during singing (Fee et al., 2004; Jarvis et al., 1998). The functional profile of adult-born HVC neurons has not yet been examined, but it is possible that in zebra finches they represent a type of HVC-RA neuron that is inactive during singing yet responsive to song playbacks. If that were the case, too, in song sparrows, it would explain why they can seasonally cull and replace neurons without changing their song from one year to the next (Smith et al., 1997).

If use of new neurons affects their survival, it could provide a clue about their function. There are four examples of a relationship between pathway use and the survival of adult-born neurons: (1) singing and the canary HVC (Li et al., 2000); (2) free-ranging life style and the hippocampus of black-capped chickadees (Barnea and Nottebohm, 1994); (3) olfaction and the olfactory bulb in mice (Petreanu and Alvarez-Buylla, 2002); and (4) associative learning and the hippocampus in rats (Gould et al., 1999). Despite these precedents, the manner in which social complexity promotes new neuron survival in the HVC of adult zebra finches is not obvious: housing males in a complex social environment does not increase their singing frequency or the amount they fly around the cage (Adar et al., 2008). Therefore, another consequence of increased social complexity must be responsible for promoting neuronal survival-perhaps the diversity of calls and songs encountered keeps new HVC cells busy. Wild zebra finches live in large colonies of up to 1000 individuals (Zann, 1996). A male that is more proficient at recognizing the songs and calls of colony members with whom it has interacted may have a selective mating advantage. If HVC plays a role in vocal recognition, as suggested by Williams and Nottebohm (1985) and Margoliash (1986), then the neurons added in adulthood might improve this ability.

Neuronal replacement in the HVC of seasonal songbirds was first observed in laboratory canaries and then described in wild- 
caught song sparrows (Tramontin and Brenowitz, 1999). Our zebra finch results are so different that it would be reassuring to see them confirmed by data from free-ranging individuals. The aviary housed zebra finches from Experiment 3 lived with the same set of birds for 3 months and we reported no loss of adultborn HVC neurons during the last 2 months of this period. If zebra finches in the wild move between social groups, a changing social environment could actually promote the turnover of existing HVC neurons, as new birds and new songs need to be identified and old memories become obsolete.

Given that zebra finches accumulate adult-born neurons in HVC with little or no culling of existing cells, why do seasonal species opt to replace HVC neurons when they could keep them all? Initially, observations on canaries suggested that replacement was necessary for the motor learning of new songs, but later studies in song sparrows showed replacement in them too, even though no new songs were learnt in adulthood. It probably comes down to costs and benefits. A male zebra finch that must start breeding on very short notice when the rains arrive, must have all brain circuits for this task available at all times. A more seasonal bird might be able to economize on energy demands by partially dismantling circuits that, during half of the year, are not needed. This, in turn, may offer the opportunity to update memory banks and discard information that is no longer relevant. Life-style differences could determine how HVC best achieves efficiency.

But speculation is not knowledge. We are still far from understanding what contribution, if any, adult-born neurons make to song system function. Maybe adult-born HVC neurons have nothing to do with auditory, vocal, or social learning. Clearly, the anatomical consequences of neuronal recruitment in the HVC of adult zebra finches are markedly different from those observed in adult canaries and song sparrows. It was perplexing to discover that, in the canary HVC, neurons recruited in adulthood were transient members of existing circuits. Now, we are equally surprised by the observation that neurons recruited into the HVC of adult zebra finches are part of a net growth in numbers associated with a reduction in cell size and an increase in packing density. Both outcomes are very different and should be kept in mind when imagining what adult neurogenesis can do and how it might relate to clinical settings.

\section{References}

Adar E, Lotem A, Barnea A (2008) The effect of social environment on singing behavior in the zebra finch (Taeniopygia guttata) and its implication for neuronal recruitment. Behav Brain Res 187:178-184.

Airey DC, Castillo-Juarez H, Casella G, Pollak EJ, DeVoogd TJ (2000) Variation in the volume of zebra finch song control nuclei is heritable: developmental and evolutionary implications. Proc Biol Sci 267:2099-2104.

Alvarez-Borda B, Haripal B, Nottebohm F (2004) Timing of brain-derived neurotrophic factor exposure affects life expectancy of new neurons. Proc Natl Acad Sci U S A 101:3957-3961.

Alvarez-Buylla A, Theelen M, Nottebohm F (1988) Birth of projection neurons in the higher vocal center of the canary. Proc Natl Acad Sci U S A 85:8722-8726.

Alvarez-Buylla A, Kirn JR, Nottebohm F (1990) Birth of projection neurons in adult avian brain may be related to perceptual or motor learning. Science 249:1444-1446.

Arai O, Taniguchi I, Saito N (1989) Correlation between the size of song control nuclei and plumage color change in orange bishop birds. Neurosci Lett 98:144-148.

Aronov D, Andalman AS, Fee MS (2008) A specialized forebrain circuit for vocal babbling in the juvenile songbird. Science 320:630-634

Barami K, Iversen K, Furneaux H, Goldman SA (1995) Hu protein as an early marker of neuronal phenotypic differentiation by subependymal zone cells of the adult songbird forebrain. J Neurobiol 28:82-101.

Barnea A, Nottebohm F (1994) Seasonal recruitment of hippocampal neu- rons in adult free-ranging black-capped chickadees. Proc Natl Acad Sci U S A 91:11217-11221.

Barnea A, Mishal A, Nottebohm F (2006) Social and spatial changes induce multiple survival regimes for new neurons in two regions of the adult brain: an anatomical representation of time? Behav Brain Res 167:63-74.

Bernard DJ, Ball GF (1995) Two histological markers reveal a similar photoperiodic difference in the volume of the high vocal center in male European starlings. J Comp Neurol 360:726-734.

Bottjer SW, Miesner EA, Arnold AP (1984) Forebrain lesions disrupt development but not maintenance of song in passerine birds. Science 224:901-903.

Brenowitz EA (2004) Plasticity of the adult avian song control system. Ann N Y Acad Sci 1016:560-585

Brenowitz EA, Nalls B, Wingfield JC, Kroodsma DE (1991) Seasonal changes in avian song nuclei without seasonal changes in song repertoire. J Neurosci 11:1367-1374.

Brenowitz EA, Baptista LF, Lent K, Wingfield JC (1998) Seasonal plasticity of the song control system in wild Nuttall's white-crowned sparrows. J Neurobiol 34:69-82.

Fee MS, Kozhevnikov AA, Hahnloser RH (2004) Neural mechanisms of vocal sequence generation in the songbird. Ann NY Acad Sci 1016:153-170.

Geinismann YY, Larina VN, Mats VN (1971) Changes of neurones dimensions as a possible morphological correlate of their increased functional activity. Brain Res 26:247-257.

Goldman SA, Nottebohm F (1983) Neuronal production, migration and differentiation in a vocal control nucleus of the adult female canary brain. Proc Natl Acad Sci U S A 80:2390-2394.

Gould E, Beylin A, Tanapat P, Reeves A, Shors TJ (1999) Learning enhances adult neurogenesis in the hippocampal formation. Nat Neurosci 2:260-265.

Gundersen HJ, Jensen EB (1987) The efficiency of systematic sampling in stereology and its prediction. J Microsc 147:229-263.

Hahnloser RH, Kozhevnikov AA, Fee MS (2002) An ultra-sparse code underlies the generation of neural sequences in a songbird. Nature 419: $65-70$.

Herrmann K, Arnold AP (1991) The development of afferent projections to the robust archistriatal nucleus in male zebra finches: a quantitative electron microscopic study. J Neurosci 11:2063-2074.

Hessler NA, Doupe AJ (1999) Social context modulates singing-related neural activity in the songbird forebrain. Nat Neurosci 2:209-211.

Immelmann K (1969) Song development in the zebra finch and other estrilded finches. Bird vocalization. Cambridge, UK: Cambridge UP.

Jarvis ED, Scharff C, Grossman MR, Ramos JA, Nottebohm F (1998) For whom the bird sings: context-dependent gene expression. Neuron 21:775-788.

Kao MH, Brainard MS (2006) Lesions of an avian basal ganglia circuit prevent context-dependent changes to song variability. J Neurophysiol 96:1441-1455.

Katz LC, Gurney ME (1981) Auditory responses in the zebra finch's motor system for song. Brain Res 221:192-197.

Katz LC, Burkhalter A, Dreyer WJ (1984) Fluorescent latex microspheres as a retrograte neuronal marker for in vivo and in vitro studies of visual cortex. Nature 310:498-500.

Kirn JR, Nottebohm F (1993) Direct evidence for loss and replacement of projection neurons in adult canary brain. J Neurosci 13:1654-1663.

Kirn JR, Alvarez-Buylla A, Nottebohm F (1991) Production and survival of projection neurons in a forebrain vocal center of adult male canaries. J Neurosci 11:1756-1762.

Kirn J, O'Loughlin B, Kasparian S, Nottebohm F (1994) Cell death and neuronal recruitment in the high vocal center of adult male canaries are temporally related to changes in song. Proc Natl Acad Sci U S A 91:7844-7848.

Kirn JR, Fishman Y, Sasportas K, Alvarez-Buylla A, Nottebohm F (1999) Fate of new neurons in adult canary high vocal center during the first 30 days after their formation. J Comp Neurol 411:487-494.

Li XC, Jarvis ED, Alvarez-Borda B, Lim DA, Nottebohm F (2000) A relationship between behavior, neurotrophin expression, and new neuron survival. Proc Natl Acad Sci U S A 97:8584-8589.

Lipkind D, Nottebohm F, Rado R, Barnea A (2002) Social change affects the survival of new neurons in the forebrain of adult songbirds. Behav Brain Res 133:31-43.

Margoliash D (1983) Acoustic parameters underlying the responses of 
song-specific neurons in the white-crowned sparrow. J Neurosci 3:1039-1057.

Margoliash D (1986) Preference for autogenous song by auditory neurons in a song system nucleus of the white-crowned sparrow. J Neurosci 6:1643-1661.

Marler P, Peters S (1987) A sensitive period for song acquisition in the song sparrow, Melospiza Melodia: a case of age-limited learning. Ethology $76: 89-100$.

Mooney R (2000) Different subthreshold mechanisms underlie song selectivity in identified HVc neurons of the zebra finch. J Neurosci 20:5420-5436.

Nixdorf BE, Davis SS, DeVoogd TJ (1989) Morphology of Goldiimpregnated neurons in hyperstriatum ventralis, pars caudalis in adult male and female canaries. J Comp Neurol 284:337-349.

Nordeen KW, Nordeen EJ (1988) Projection neurons within a vocal motor pathway are born during song learning in zebra finches. Nature 334:149-151.

Nottebohm F (1981) A brain for all seasons: cyclical anatomical changes in song control nuclei of the canary brain. Science 214:1368-1370.

Nottebohm F (1985) Neuronal replacement in adulthoon. Ann N Y Acad Sci 457:143-161.

Nottebohm F, Nottebohm ME, Crane L (1986) Developmental and seasonal changes in canary song and their relation to changes in the anatomy of song-control nuclei. Behav Neural Biol 46:445-471.

Olveczky BP, Andalman AS, Fee MS (2005) Vocal experimentation in the juvenile songbird requires a basal ganglia circuit. PLoS Biol 3:e153.

Paton JA, Nottebohm FN (1984) Neurons generated in the adult brain are recruited into functional circuits. Science 225:1046-1048.

Petreanu L, Alvarez-Buylla A (2002) Maturation and death of adult-born olfactory bulb granule neurons: role of olfaction. J Neurosci 22:6106-6113.

Pytte CL, Gerson M, Miller J, Kirn JR (2007) Increasing stereotypy in adult zebra finch song correlates with a declining rate of adult neurogenesis. Dev Neurobiol 67:1699-1720.

Scharff C, Nottebohm F (1991) A comparative study of the behavioral deficits following lesions of various parts of the zebra finch song system: implications for vocal learning. J Neurosci 11:2896-2913.

Scharff C, Kirn JR, Grossman M, Macklis JD, Nottebohm F (2000) Targeted neuronal death affects neuronal replacement and vocal behavior in adult songbirds. Neuron 25:481-492.

Scott BB, Lois C (2007) Developmental origin and identity of song system neurons born during vocal learning in songbirds. J Comp Neurol 502:202-214.

Scotto-Lomassese S, Rochefort C, Nshdejan A, Scharff C (2007) HVC in- terneurons are not renewed in adult male zebra finches. Eur J Neurosci 25:1663-1668.

Smith GT, Brenowitz EA, Wingfield JC, Baptista LF (1995) Seasonal changes in song nuclei and song behavior in Gambel's white-crowned sparrows. J Neurobiol 28:114-125.

Smith GT, Brenowitz EA, Beecher MD, Wingfield JC (1997) Seasonal changes in testosterone, neural attributes of song control nuclei, and song structure in wild songbirds. J Neurosci 17:6001-6010.

Tramontin AD, Brenowitz EA (1999) A field study of seasonal neuronal incorporation into the song control system of a songbird that lacks adult song learning. J Neurobiol 40:316-326.

Vates GE, Broome BM, Mello CV, Nottebohm F (1996) Auditory pathways of caudal telencephalon and their relation to the song system of adult male zebra finches. J Comp Neurol 366:613-642.

Vellema M, van der Linden A, Gahr M (2010) Area-specific migration and recruitment of new neurons in the adult songbird brain. J Comp Neurol 518:1442-1459.

Wang N, Hurley P, Pytte C, Kirn JR (2002) Vocal control neuron incorporation decreases with age in the adult zebra finch. J Neurosci 22:10864-10870

Ward BC, Nordeen EJ, Nordeen KW (2001) Anatomical and ontogenetic factors producing variation in HVc neuron number in zebra finches. Brain Res 904:318-326.

West MJ (1993) New Stereological methods for counting neurons. Neurobiol Aging 14:275-285.

West MJ, Gundersen HJ (1990) Unbiased stereological estimation of the number of neurons in the human hippocampus. J Comp Neurol 296:1-22.

Wilbrecht L, Williams H, Gangadhar N, Nottebohm F (2006) High levels of new neuron addition persist when the sensitive period for song learning is experimentally prolonged. J Neurosci 26:9135-9141.

Wild JM (1997) Functional anatomy of neural pathways contributing to the control of song production in birds. Eur J Morphol 35:303-325.

Wild JM, Williams MN, Howie GJ, Mooney R (2005) Calcium-binding proteins define interneurons in HVC of the zebra finch (Taeniopygia guttata). J Comp Neurol 483:76-90.

Williams H, Nottebohm F (1985) Auditory responses in avian vocal motor neurons: a motor theory for song perception in birds. Science 229:279-282.

Woolley SC, Doupe AJ (2008) Social context-induced song variation affects female behavior and gene expression. PLoS Biol 6:e62.

Zann R (1996) The zebra finch: synthesis of field and laboratory studies. New York: Oxford UP. 